\title{
Quantifying local characteristics of velocity, aggregation and hematocrit of human erythrocytes in a microchannel flow
}

\author{
Efstathios Kaliviotis $^{\mathrm{a}, \mathrm{c}, *}$, Jonathan Dusting $^{\mathrm{b}}$, Joseph M. Sherwood ${ }^{\mathrm{b}}$ and Stavroula Balabani ${ }^{\mathrm{c}}$ \\ ${ }^{a}$ Department of Mechanical Engineering and Materials Science and Engineering, Cyprus University \\ of Technology, Limassol, Cyprus, UK \\ ${ }^{\mathrm{b}}$ Department of Bioengineering, Imperial College London, UK \\ ${ }^{\mathrm{c}}$ Department of Mechanical Engineering, University College London, UK
}

\begin{abstract}
The effect of erythrocyte aggregation on blood viscosity and microcirculatory flow is a poorly understood area of haemodynamics, especially with relevance to serious pathological conditions. Advances in microfluidics have made it possible to study the details of blood flow in the microscale, however, important issues such as the relationship between the local microstructure and local flow characteristics have not been investigated extensively. In the present study an experimental system involving simple brightfield microscopy has been successfully developed for simultaneous, time-resolved quantification of velocity fields and local aggregation of human red blood cells (RBC) in microchannels. RBCs were suspended in Dextran and phosphate buffer saline solutions for the control of aggregation. Local aggregation characteristics were investigated at bulk and local levels using statistical and edge-detection image processing techniques. A special case of aggregating flow in a microchannel, in which hematocrit gradients were present, was studied as a function of flowrate and time. The level of aggregation was found to strongly correlate with local variations in velocity in both the bulk flow and wall regions. The edge detection based analysis showed that near the side wall large aggregates are associated with regions corresponding to high local velocities and low local shear. On the contrary, in the bulk flow region large aggregates occurred in regions of low velocity and high erythrocyte concentration suggesting a combined effect of haematocrit and velocity distributions on local aggregation characteristics. The results of this study showed that using multiple methods for aggregation quantification, albeit empirical, could help towards a robust characterisation of the structural properties of the fluid.
\end{abstract}

Keywords: Blood flow, cell aggregation, micro-PIV, image processing techniques

\section{Introduction}

Hyperviscosity, an increase in blood viscosity from the physiological state, is associated with a large number of pathological conditions, such as sickle cell anaemia [2], arterial hypertension [25] and Diabetes Mellitus [19]. However, its role in vascular resistance remains unclear; although hyperviscosity increases flow resistance locally, it can induce production of vasodilators such as nitric oxide in response to higher shear stresses acting on the endothelium. This results in an increase in the luminal diameter and thus

\footnotetext{
${ }^{*}$ Corresponding author: Efstathios Kaliviotis, Department of Mechanical Engineering and Materials Science and Engineering, Cyprus University of Technology, Limassol, 3041 Cyprus, UK. Tel.: +357 25002289; Fax: +357 25002637; E-mail: e.kaliviotis@cut.ac.cy.
} 
reduces flow resistance [47]. In order to improve understanding of the local and systemic effects, the mechanical factors affecting blood viscosity must be fully considered.

Blood is a multi-phase fluid consisting of a suspension of erythrocytes (red blood cells), leukocytes and platelets in a continuous fluid phase (plasma). Erythrocytes account for $99 \%$ of the formed elements and thus greatly affect the fluid mechanical properties of blood. In large vessels (arteries and veins), blood is generally considered to behave as a Newtonian fluid due to the high shear rates developed in the flow. However, experiments on pressure drop and flow in microvessels suggest that an increase in viscosity with a decrease in shear should be expected as a result of the erythrocyte aggregation and other factors [34]; this is due to the shear thinning nature of blood (decrease of viscosity with the increase of shear rate). According to the depletion model of aggregation [39], an osmotic gradient occurs between erythrocytes in close proximity due to local depletion of plasma proteins, which draws the cells together. Another explanation of the aggregation process is given by the bridging model, which states that aggregation is the result of neighboring RBCs absorbing the same macromolecular chain [9]. The deformability of RBCs is an important parameter influencing the flow of blood; it is partly responsible for the non-Newtonian nature of blood and it plays a role in the intensity of aggregation [45]. Disaggregation occurs when shear forces overcome the aggregating forces, i.e. it occurs increasingly at higher shear rates. Aggregation enhances the tendency of erythrocytes to axially migrate, while also blunting the time-averaged velocity profile. The competition between these two effects has, depending on other factors, been shown to result in either an increase $[11,46]$ or decrease $[4,35,56]$ in the apparent viscosity. Bishop et al. [5] observed, using in vivo imaging of rat venules, that cell aggregation slightly reduced the average variability of red blood cell velocity and radial position, although this reduction compared to the overall standard deviation. Additionally, erythrocyte aggregation has been shown to be associated with a decrease in erythrocyte velocity in cutaneous capillaries in diabetic patients [21]. In order to further understand these phenomena, more detailed quantification on the effect of aggregation under controlled flow conditions is required.

There are several techniques available for measuring blood flow, of which Particle Image Velocimetry (PIV) is the most suitable for applications where small scales and high spatial resolution are required [59]. PIV is an image-based, full-field velocity measurement technique in which pairs of particle images are divided into interrogation windows and correlated. The result represents the most probable average velocity of the particles (or tracer objects) within the interrogation window. Micro-PIV is a variation of the technique designed to measure microscale flows, developed based on the pioneering work of Santiago et al. [48], Koutsiaris et al. [27] and Meinhart et al. [37, 38]. A comprehensive description of the technique can be found in [28]. Micro-PIV has been applied to blood flows in vitro [20, 29, 31, 41, 42] and in vivo $[12,43,49,57,58]$. In these studies, either the flow is seeded with fluorescent particles or the erythrocytes themselves are used as tracer particles. A review of other microscopic techniques for the measurement of erythrocyte velocities in vivo is provided in [30].

Aggregation measurement is generally carried out separately to flow experiments, with the extent of aggregation indirectly quantified by the level of light transmitted or backscattered by samples, although several other measures have been employed. Typically these techniques are defined based on transient responses and threshold shear rates, and with measurements taking place in controlled rotational systems. However, as aggregation is shear dependent, the information collected from such systems is not directly applicable to channel and more complex flows, where the shear distribution differs significantly. Shin et al. [54] developed a method based on laser back-scattering in a disposable microchannel. However, to the authors' knowledge, aggregation has not been studied in combination with accurate measurement of the flow field within microchannels, such as that acquired using PIV. Recent micro-PIV studies by Sherwood et al. $[50,51]$ reported in detail on the effect of erythrocyte aggregation on various aspects of 
blood flow, such as cell depletion, flow field and viscosity. In these studies however, the local structural characteristics of blood were not quantified, and therefore their relationship with the local flow within the microchannel was not investigated.

For quantifying the complex interactions between the velocity field and aggregation, there is a need for simultaneous measurement of the two parameters. Dusting et al. [13] described a method for yielding both velocity data and a bulk aggregation index from images of erythocytes in a plate-plate shearing system at physiological and uniformly distributed haematocrit conditions. The characteristics of RBC aggregation in conditions similar to these, were quantified also in a subsequent study by Kaliviotis et al. [22], and in combination with the derived shearing fields the spatial variation of blood viscosity was determined. In the present study, a newly developed technique is employed to simultaneously measure local aggregation and the velocity fields, corresponding to different erythocyte aggregation states, for blood flowing in microchannels. This is a significant improvement from the study of Kaliviotis et al. [22] in that aggregation is measured in the local level (in the scale of cells and aggregates), and in the presence of hematocrit and velocity gradients in a rectangular microchannel. Microchannel RBC flows are useful for studying aggregating blood flows as they provide more realistic hematocrit and velocity gradients, exhibit cell depletion layers, and are a common feature of microdevices. PIV measurements reported herein are achieved without a laser, fluorescent particles, or a dichroic mirror. The technique is used to describe flow characteristics in relation to local structural aspects as they change due to erythrocyte aggregation. In particular, this work is to the authors knowledge the first in which local aggregation characteristics are studied in relation to the velocity distribution in a microchannel, with the purpose of providing detailed information on human blood flow dynamics in more complex microchannel geometries; it is demonstrated that the utilisation of two image processing techniques for characterising the extent of aggregation in a micro channel has the potential to capture the local microstructure characteristics.

\section{Methodology}

\subsection{Sample preparation}

The study was approved by the South East London Research Ethics Committee (ref: 10/H0804/21) and proper informed consent was obtained from volunteers. Approximately $18 \mathrm{ml}$ of flood was collected from healthy volunteers into vacuum tubes (BD, 367873) preloaded with $1.8 \mathrm{mg} / \mathrm{ml}$ EDTA to prevent coagulation. The erythrocytes were washed twice in Phosphate Buffered Saline (PBS), centrifuged at 3600 rpm, and resuspended in PBS containing Dextran 2000 (5 g/l) (referred to as D2000 cases hereafter also). Dextran is a family of neutral polymers that according to their molecular weight and concentration influence the intensity of the RBC aggregation phenomenon [9]. The particular molecular weight (2000 $\mathrm{KDa}$ ) and concentration was chosen to induce a moderate aggregation tendency, according to the viscosity and aggregation curves found in [9]. Additional baseline experiments were conducted without Dextran 2000. The haematocrit (erythrocyte concentration by volume) was $25 \%$, which is within the range of physiological values for microvessels with sizes similar to the models used in this study. For consistency all experiments were conducted with a single blood sample.

\subsection{Flow system}

Custom SU-8 microchannels fabricated directly into SU-8 chips with photolithography (Epigem, Redcar, UK), were mounted on an inverted microscope and connected to a simple microfluidic system, 


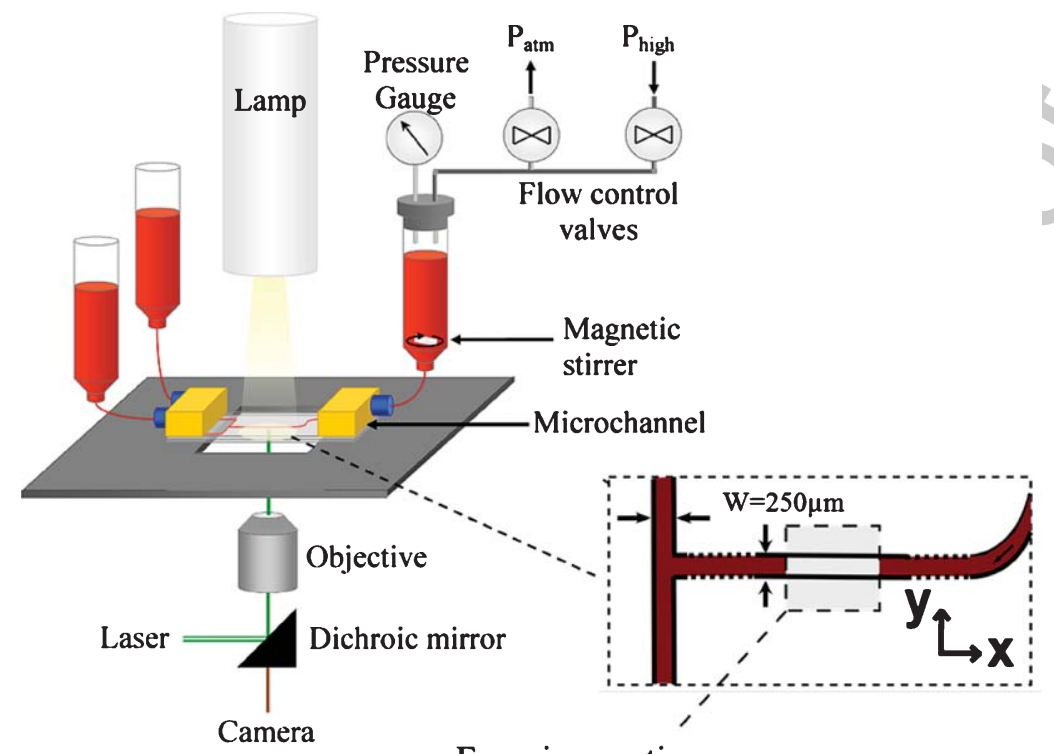

Focusing section

Fig. 1. Annotated schematic of the microchannel flow, with brightfield micro-PIV measurement system: the width and depth of the channel were $W=250 \mu \mathrm{m}$ and $D=50 \mu \mathrm{m}$, respectively (detailed section not shown in scale). The distance from the focusing section to the curved inlet section was $2.5 \mathrm{~cm}$ and the radius of curvature approximately $1 \mathrm{~cm}$.

as shown in Fig. 1. As a material, SU-8 is a versatile epoxy-based, negative tone photoresist, which is sensitive to radiation and ideal for the construction of rigid, high aspect ratio microchannels [1]. The microchannel consisted of a curved section followed by a long straight section, and finally a T-bifurcation. The width and depth of the channel in all regions were $W=250 \mu \mathrm{m}$ and $D=50 \mu \mathrm{m}$, respectively. The distance from the focusing region to the T-junction and curved sections was $2.5 \mathrm{~cm}(300 \times$ the hydraulic diameter) and the radius of curvature was approximately $1 \mathrm{~cm}$. Therefore, the flow was considered as fully developed, as the recovery length was estimated to be very small $(\approx 1 \times$ the channel width). The sample was perfused through the microchannel by generating a pressure drop between the sealed inlet reservoir and the open outlet reservoirs. The air pressure in the inlet reservoir was controlled with a needle valve, which led to more stable flow and faster response times than directly pumping the sample with a syringe or peristaltic pump. The reservoir pressure, and therefore the pressure drop across the channel, was varied to obtain velocities similar to those occurring in the human microcirculation [14] and a range of aggregation conditions.

Flow rates were not measured directly but were estimated based on the measured values in the velocity profile averaged over the axial $(x)$ domain, and the two-dimensional analytical solution for a Newtonian fluid in laminar flow in a rectangular channel [7]. The flow rates produced were in the range of 2.84 to $69.6 \mu l / h(78.9$ to $1933.3 \mathrm{nl} / \mathrm{s})$ for the samples treated with Dextran and 3.5 to $61 \mu l / h(97.2$ to $1694.5 \mathrm{nl} / \mathrm{s}$ ) for the RBCs suspended in PBS.

The corresponding pseudo-shear rates were calculated as $\dot{\gamma}=U / h$, with $U$ being the mean velocity calculated from the flow rate and $h$ the height of the channel; they were found to be in the range of 0.5 to $31.0 \mathrm{~s}^{-1}$ for the Dextran samples and 1.6 to $27.3 \mathrm{~s}^{-1}$ for the PBS cases. It should be noted that the pseudoshear rate is used as a rough measure of the flow strength in this study which is common practice in the literature. Although the use of pseudoshear rate is appropriate in Couette type of flows, its use in 
rectangular microchannels, should be made with caution: in a high aspect ratio like the one in the present study the pseudoshear values are approximately half of those calculated for a single phase, Newtonian fluid using depth averaging of the shear magnitude field. To develop a more meaningful nominal shear rate index the channel geometry, the effects of aggregation on the structure of the fluid (such as plug-flow at certain conditions) and the optical properties of the microscopy system (mainly depth of focus) should be taken into consideration.

In order to minimise separation of erythrocytes from the bulk flow, which is especially important for aggregating samples, cells were disaggregated between cases by increasing the flowrate, tube lengths were minimised, and the fluid in the inlet reservoir was continuously mixed with a magnetic stir bar. No damage was observed to cells at the flowrates used; cells were frequently visually inspected under the microscope for shape changes, and the color of the suspending medium was visually and optically (through inspection of the intensity values of plasma) monitored for indications of hemolysis in the sample. All experiments were conducted at room temperature $\left(25^{\circ} \mathrm{C}\right)$.

The tube haematocrit was estimated to be $\sim 20 \%$ using a conversion factor based on the data of Barbee and Cokelet [3] and an hydraulic diameter of $83 \mu \mathrm{m}$. The data presented in the this work show that there is a non-uniform distribution of the RBCs in the cross-stream direction. This was mainly due to the curved inlet section of the microchannel; cells experience lift forces close to boundaries due to velocity and shear rate gradients. As those gradients are different in the opposite walls of the curved section, cells tend to translate towards the side of the favorable gradient. This mechanism is used in micro-devices for cell separation (see for example the studies of [6] and [18]). Nevertheless, the data were deemed particularly suitable for an in depth analysis of the relationship of the microstructure of the fluid and flow velocities, with the purpose of validating a local aggregation measurement technique.

\subsection{Brightfield micro-PIV data acquisition and processing}

Micro-PIV experiments were performed using brightfield illumination with a $10 X$ objective $(\mathrm{NA}=0.25)$ and then correlating the image patterns produced by erythrocytes. The methodology followed was similar to that previously described by Dusting et al. [13] and Sherwood et al. [50]. Images of $1280 \times 384$ pixels, which covered an area of $w \times 3.3 w$, were recorded using a IDT X3 CMOS camera (Tallahassee, USA) at a spatial resolution of $0.65 \mu \mathrm{m} /$ pixel. The camera uses a 1.3 MPixel sensor, of 4:3 aspect ratio, with a $12 \times 12 \mu \mathrm{m}$ pixel size, a pixel fil factor of $40 \%$ and a quantum efficiency of $\approx 40 \%$. A $100 \mathrm{~W}$ halogen white light source was used to illuminate the imaged region of the channel from above. For each flowrate, a set of data was acquired every 2 seconds for 60 s after adjusting the flowrate. For each data set, 100 images of $100 \mu \mathrm{s}$ exposure were acquired at a frame rate that was adjusted to optimise the time interval between image pairs $(\Delta t)$. Images were processed using the open source software JPIV (http://www.jpiv.vennemann-online.de) with an interrogation window size of $32 \times 32$ pixels and with an overlap of $50 \%$. Fewer than $0.3 \%$ of vectors were detected to be invalid by a normalised median test according to Westerweel and Scarano [60]; this test utilises the median of residuals of 8 neighbouring vectors to normalise the displacement vector in question. After the normalisation a threshold can be set as the limit of validity ( 2.0 in the present study). Invalidated vectors were replaced with median filtered values.

The depth of focus of a system with the above-mentioned characteristics is expected to be relatively large: it is estimated at approximately $18 \mu \mathrm{m}$ when calculated as in [27], using extreme values for wavelength $(\lambda \approx 700 \mathrm{~nm})$ and 1.6 as the refractive index of the SU8 microchannel. The depth of focus is estimated even larger $(\approx 38 \mu \mathrm{m})$ when referring to more advanced methods [40]. In addition, the nature of the fluid, which is a dense suspension of biconcave RBCs and, at low shearing flows, aggregates, further 


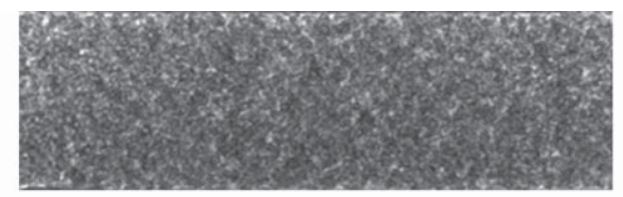

(a)

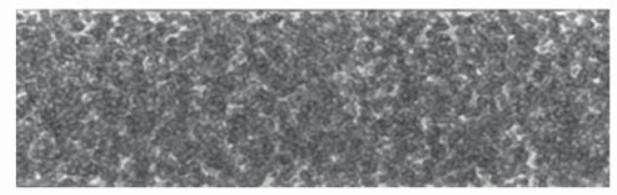

(b)

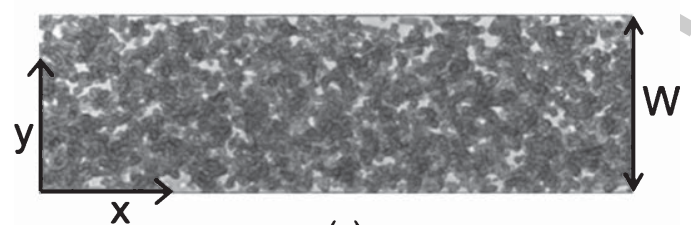

(c)

Fig. 2. Brightfield images of erythrocyte suspensions suspended in a Dextran solution flowing through the microchannel. (a) $Q=69.6 \mu \mathrm{l} / \mathrm{h}\left(31.0 s^{-1}\right)$ (no aggregation); (b) $Q=19.0 \mu \mathrm{l} / \mathrm{h}\left(8.5 s^{-1}\right)$ (moderate aggregation) and (c) $Q=2.84 \mu \mathrm{l} / \mathrm{h}\left(1.3 s^{-1}\right)$ (high aggregation). Flow direction is from right to left. Note that magnification is the same for all images.

contributes to the large depth of focus, which is expected to engulf the largest part of the channel depth. Nevertheless, although in $\mu m P I V$ techniques the large depth of focus influences the velocity results, data produced from such processing are still very useful for the analysis of the in-plane velocity field as a mean quantity.

The brightfield illuminated micro-PIV technique has been validated in a previous study by Sherwood et al. [51]. In that study, erythrocyte velocities were compared with the continuous phase velocity fields, measured using fluorescent microscopic particle image velocimetry, and with the velocity fields obtained by using computational fluid dynamics. The overall error in the micro-PIV measurements has been estimated to $3.5 \%$, for the non-aggregating cases [52]. The limitations of both the brightfield and the fluorescence $\mu$ PIV techniques were discussed in detail in the aforementioned studies.

Images of erythrocytes, suspended in a Dextran solution, acquired for three selected flow cases are shown in Fig. 2. All were captured as part of image sets approximately $60 \mathrm{~s}$ after adjusting the flow. Fig. 2(a) corresponds to $Q=69.6 \mu \mathrm{l} / \mathrm{h}\left(31.0 \mathrm{~s}^{-1}\right)$, the highest flowrate and pseudo shear rate investigated, and represents a state in which the erythrocytes are completely disaggregated and visible as individual cells. For $Q=19.0 \mu \mathrm{l} / \mathrm{h}\left(8.5 s^{-1}\right)$, shown in Fig. 2(b), erythrocyte aggregation is apparent, with further aggregation occurring at $Q=2.84 \mu \mathrm{l} / \mathrm{h}\left(1.3 s^{-1}\right)$ (Fig. 2(c)). This trend is expected as the shear rate magnitudes, while varying throughout the channel, scale closely with $Q$, and aggregation is associated with lower shear rates.

\subsection{Red blood cell aggregation measurements - Image processing techniques}

The extent of aggregation can be described indirectly via the measurement of a number of different parameters (e.g. settling times, light transmission, imaging techniques). In this work two different 
approaches, both based on image processing, have been adopted. The software used for the processing of the monochromatic images was MATLAB (Mathworks), together with functions developed by Gonzales, et al. [17].

\subsubsection{Image contrast method}

The first method is based on the fact that image contrast is increased as a result of cell aggregation. This is because gaps between erythrocytes caused by aggregation lead to a greater transmission of light through the channel, while regions where there are dense aggregates lead to locally enhanced light absorption. The parameter used to describe the variation of contrast was the mean standard deviation of intensity $\left(\sigma_{I^{*}}\right)$ in the cross-stream direction $(y)$. The calculated values of $\sigma_{I^{*}}$ where averaged over the entire image in the direction of flow $(x)$. This measure provided an index describing the extent of aggregation within the whole region under consideration.

\subsubsection{Edge detection method}

The second method was developed taking advantage of the image properties of RBC aggregates. More specifically, connected RBCs (rouleaux) result in continuous isointensity patterns, which are detectable with appropriate edge detection algorithms; these continuous areas of similar intensity increase with the increase in the number of cells per rouleaux. To model accurately the dependency of the isointensity areas on the number of RBCs, and consequently to model the increase in aggregate size, is not a trivial task; for instance, depending on the orientation of a rouleaux, captured in the two-dimensional image, isointensity patterns of different size may develop. In the simplest case where an evolving rouleaux is captured sideways in the image the isointensity areas should increase linearly with the increase of the number of RBCs per rouleaux. Larger aggregates, however, are more complex structures which result from the combination of rouleaux. The basic and important steps of the image processing are explained bellow.

Step 1 . The preprocessing of the images was kept at minimum in order to preserve the local characteristics of the developed features in the fluid. Initially, an intensity transformation was performed in order to expand the intensity range of the image, and increase the image contrast; this was achieved utilising a contrast-stretching algorithm developed by Gonzales, et al. [17].

Step 2 . The magnitude of the local intensity gradients $\left(\nabla I_{i}\right)$ were detected using an algorithm based on the Prewit method [17]: four intensity differences per unit space $\left(\frac{\Delta I}{\Delta S}\right)$ from all eight oppositeneighbouring pixels were computed and then the magnitude of the local intensity gradient was calculated by applying spatial convolution:

$$
\nabla I_{i}=\sqrt{\frac{\Delta I_{(i, j+1)-(i, j-1)}^{2}}{\Delta S^{2}}+\frac{\Delta I_{(i+1, j)-(i-1, j)}^{2}}{\Delta S^{2}}+\frac{\Delta I_{(i+1, j+1)-(i-1, j-1)}^{2}}{\Delta S^{2}}+\frac{\Delta I_{(i+1, j-1)-(i-1, j+1)}^{2}}{\Delta S^{2}}}
$$

$\Delta S=1$ for the horizontal and vertical neighbouring points, and $\Delta S=\sqrt{2}$ for the diagonal neighbouring points.

Step 3. The magnitude of the local gradient $\left(\bar{I}_{i}\right)$ was normalised by the mean intensity of the convolved region $\left(\bar{I}_{c o n}\right)$; this was necessary because the gradients produced by aggregates are influenced by the background intensity. In addition, this dependency of the local gradient magnitude to background intensity was found to be non-linear (see Fig. 3) due to slight differences of the mean and the modal value (the most frequent intensity value) of the data; to improve the performance of the technique the local mean intensity values were moderated by the mode $\left(M_{I}\right)$ of the whole image: 


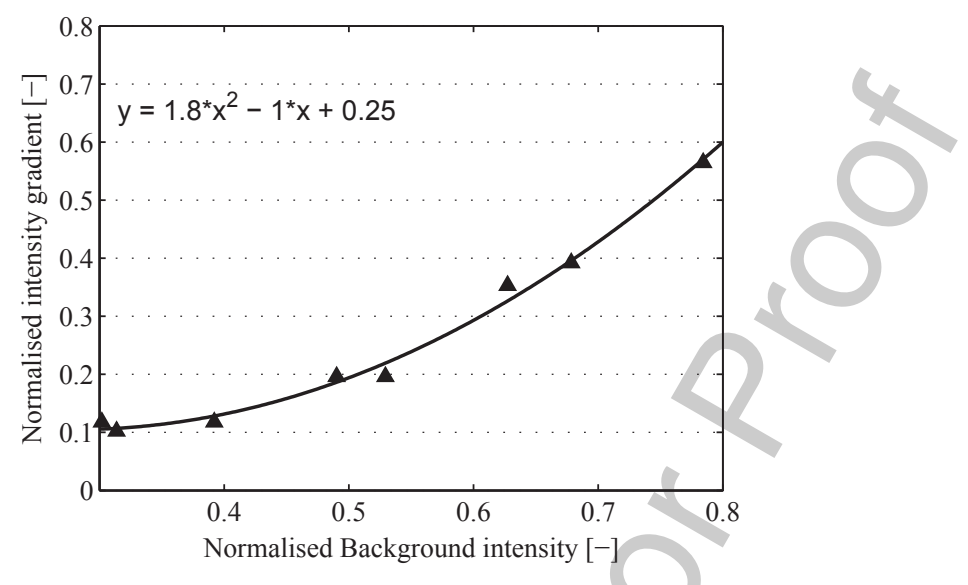

Fig. 3. The influence of the background intensity on the gradients produced by the edges of RBCs. The background intensity (mean value) was calculated from a region of $\approx 100 \times$ the $\mathrm{RBC}$ area within an image; in this particular area, the local gradient was calculated for a RBC edge in focus. The mean local background intensity and the local gradient values where normalised by the maximum intensity value in the image. The norm of fit was found to be 0.041 .

$$
E d g e=\frac{\nabla I_{i}}{\bar{I}_{c o n} M_{I}^{*}}
$$

were $M_{I}^{*}$ is the image mode normalised by the mode of a representative image from the non-aggregating cases.

Step 4. The regions in which the local gradients were above a certain threshold value were defined as edge points and converted to white (assigned a value of 1). Pixels with values below the threshold were assigned a value of 0 . Calibration of the gradient threshold value was performed in a low shear non-aggregating case and used blindly in the rest of the aggregation cases: it involved the identification of a threshold value that would result at a mean edge area (interconnected white pixels) equal to the expected edge area of one RBC. For the resolution of the current system the expected mean area of a $\mathrm{RBC}$ edge was estimated by to be $\approx 100$ pixels.

Step 5 . The detected edges (defined by the interconnected white pixels) that were smaller than $20 \%$ of the area of one RBC edge were discarded as noise.

Step 6. Statistical information about the identified edges that appeared in the processed image is extracted for further calculations and analysis: total number, area, location of edges, etc.

\subsection{Validation of the image processing techniques}

Limitations in the digital analysis of the images are mainly imposed mainly by a) the relatively large concentration of the cells in combination with the depth of the microchannel, which was 6 times the RBC diameter and b) the large depth of focus of the microscopy system, which expands in the whole gap of the microchannel. The first aspect, results in overlapping cells and aggregates (see for example Fig. 2), which in combination with the large depth of focus affect the accuracy of the edge detection technique. For this reason the applicability of image analysis techniques for the sizing of aggregates in the literature is limited to very low RBC concentrations (below 1\% in [10], [36] and [53]) or to static flow conditions for higher RBC concentrations in very small gaps [8]. The general validation process in such studies involves 


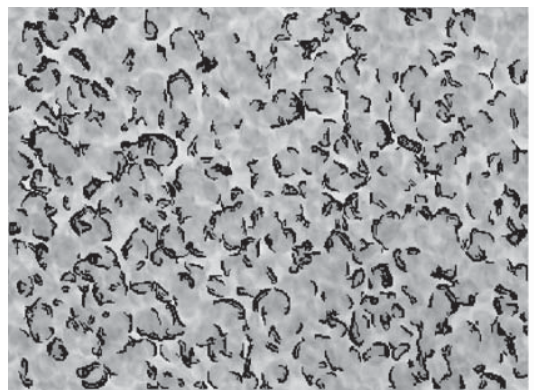

(a)

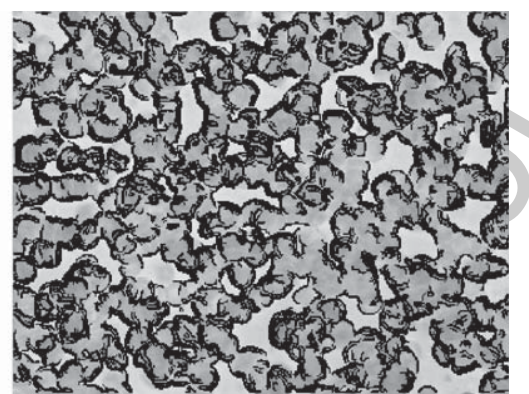

(b)

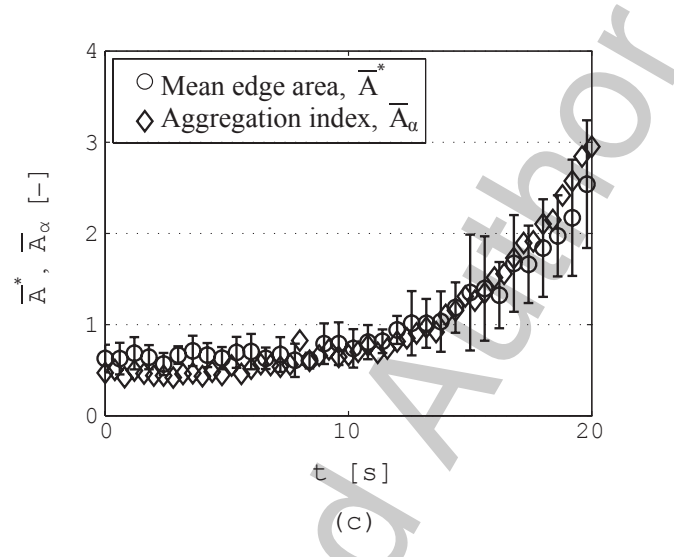

Fig. 4. Top panel: Selected images showing detected edges (black pixels) from a high shear rate $\left(\dot{\gamma}=128 \mathrm{~s}^{-1}\right.$ at $\left.t=0 \mathrm{~s}\right)(\mathrm{a})$, and a low shear rate $\left(\dot{\gamma}<1 \mathrm{~s}^{-1}\right.$ at $\left.t=20 \mathrm{~s}\right)$ (b). (c): aggregation index and edge normalised mean area $\left(\bar{A}_{\alpha}=A_{\alpha} / A(t)_{\alpha \text { mean }}\right.$ and $\bar{A}^{*}=A^{*} / A(t)_{\text {mean }}^{*}$ respectively ). Standard deviation bars are shown for the $\bar{A}^{*}$ index.

applying a method on images where the number of cells per aggregate can be counted by an operator and correlating the results. This condition does not apply on data taken at high RBC concentrations, including the data of the present study, in which the number of RBCs per aggregate cannot be resolved accurately. In order to validate the technique in the present study three different approaches were utilised and described below.

\subsubsection{Comparison of the method against other techniques}

The mean area of the detected edges $\left(\bar{A}^{*}\right)$ was compared against an aggregation index $A_{\alpha}$, which was validated against an electrorheology-based technique in the study by Kaliviotis et al. [24] (using the exact data from that study).

Figure 4 illustrates the mean detected edge area compared to the $A_{\alpha}$ index, which is a measure of the intensity of the aggregation phenomenon. Details on the formulation of the $A_{\alpha}$ index can be found in [24]; in brief, $A_{\alpha}$ is a ratio of the free plasma area (see Fig. 4b) to the area covered by RBCs, taking into account the concentration of RBCs:

$$
A_{\alpha}=\frac{\text { Total RBC free area }}{(1-\text { Hematocrit }) \times \text { Total Area }}
$$


The data used from the study of [24] were image sequences of one blood sample subjected to a range of shear rates from 128 to $0.277 \mathrm{~s}^{-1}$ in 20 seconds. In the comparison shown in Figure $4 \mathrm{c}$, both indices have been normalized by their mean value:

$$
\begin{gathered}
\bar{A}_{\alpha}=A_{\alpha} / A(t)_{\alpha \text { mean }} \\
\bar{A}^{*}=A^{*} / A(t)_{\text {mean }}^{*}
\end{gathered}
$$

This normalization was necessary due to the difference in the nature of the indices: $A^{*}$ indicates the development of aggregates from unity, whereas $A_{\alpha}$ indicates the progress of the aggregation phenomenon from a minimum, very small, decimal value. The comparison shows that qualitatively the behaviour of $A^{*}$ follows closely that of $A_{\alpha}$, although it is apparent that the time rate of change is slightly lower for $A^{*}$ compared to $A_{\alpha}$. The standard deviation of $A^{*}$ increases with the increase of aggregate size as expected

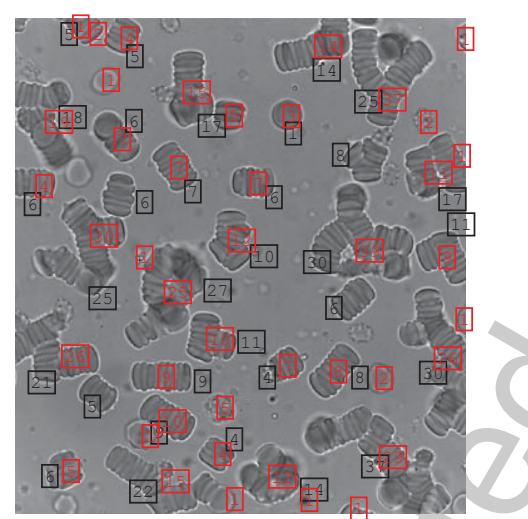

(a)

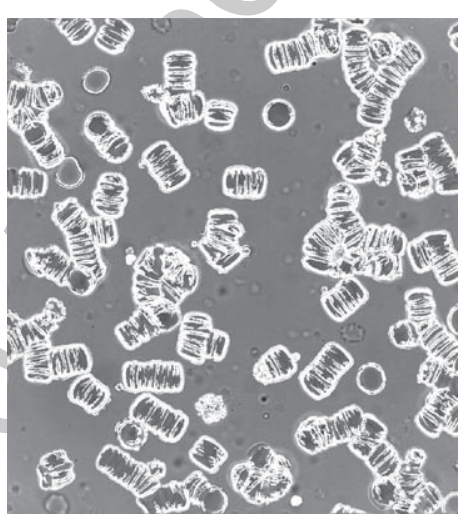

(b)

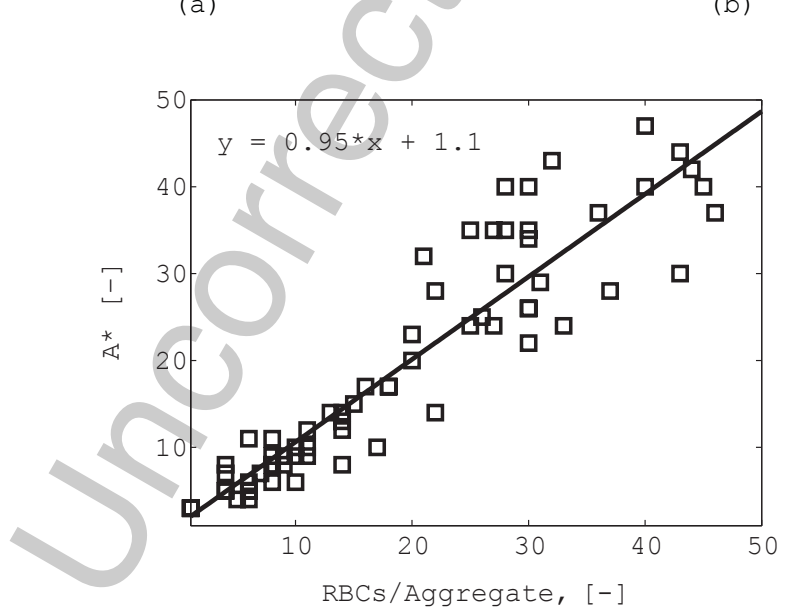

(c)

Fig. 5. Results of the second step of the validation process. (a) Image with clear aggregates, where the counted cells per aggregate are shown in black and the detected edge area in red boxes and (b) the detected edges in image (a). The correlation of $A^{*}$ to the actual number of RBCs per aggregate is shown in (c). The equation of fit show a slight underperformance of the edge detection technique for the measurement of clear aggregates, with a norm of fit at 1.6. 
due to the non-uniformity of the aggregated structures. Note that the values of $A_{\alpha}$ are not mean values and therefore no standard deviation is presented.

\subsubsection{Examination of results for images of known properties (low RBC concentrations)}

The second step of the validation process was to apply the technique on images with clear aggregates at very low $\mathrm{RBC}$ concentrations $(\approx 5 \%)$, in which the number per aggregate could be measured. The data used in this and the third step of the validation process were obtained in very small gaps $(20 \mu \mathrm{m})$ in the shearing system described in [24]. Note that $A^{*}$ is expressed as the detected edge area normalized by the edge area of one RBC, and therefore a value of one implies the edge area of one RBC. Figure 5a shows one representative image, in which the counted number of cells per aggregate are shown in black boxes and the detected values of edge areas in red boxes. The detected edge area is shown in Fig. 5b and the correlation of $A^{*}$ to the actual number of RBCs per aggregate is shown in Fig. 5c. The equation of fit shows a slight under-performance of the edge detection technique for the measurement of clear aggregates (Fig. 5 c), however the correlation coefficient indicates a reasonably good correlation ( $\mathrm{R}=0.94)$.

\subsubsection{Examination of results for images of known properties (high $R B C$ concentrations)}

For the final step of the validation process, the technique was applied on the images used in the second step after blending (averaging) those images with images of highly concentrated (45\%) dispersed RBCs, such as that in Fig. 5a. Although the image blending of the third step may result in image properties different to those encountered in a dense suspension of aggregating cells (due to averaging), the approach was utilized as an alternative due to lack of data showing clear, sizable, aggregates at high $\mathrm{RBC}$ concentrations. The results of the edge detection process for the blended image of Fig. 5a are shown in Fig. 6a (small areas are not shown for clarity) and the correlation between estimated $A^{*}$ and RBCs/aggregate counted is shown in Fig. 6(b). From the correlation it is evident that the performance of the technique deteriorates, with the norm of fit increasing at 3.4 for the blended image case $(\mathrm{R}=0.82)$.

The results in Figs. 4-6 suggest that the edge detection technique slightly underestimates the size of aggregates, and this underestimation is more pronounced for dense suspensions including larger aggregates. Nevertheless, Figs. $4 \mathrm{c}$ and $5 \mathrm{c}$ and $6 \mathrm{~b}$ show that for the purpose of studying the local structural properties of blood the current methodology could be utilized with a considerable degree of confidence for relatively small aggregates ( $<20 \mathrm{RBCs}$ per aggregate); the norm of the fit for this range was improved to 0.73 and 1.3 for the correlation of clear and blended images respectively. It should be stressed here that the blending of the images with a $45 \%$ image of dispersed RBCs performed in this study represents an extreme case; the maximum RBC concentration estimated in this study does not exceed the $30 \%$, which is the physiological concentration in the microcirculation.

\subsection{Local velocity of detected structures and shear rate magnitude}

For the analysis of the flow characteristics of the larger detected structures (see for example Fig. 5b) it was necessary to calculate a local mean velocity $\left(u_{l}\right)$ from the velocity vectors found within the area covered by the structure; this was made possible by using the coordinates of the bounding box of the detected structure. The local mean velocity $u_{l}$ was non-dimensionalised by a normalisation with the maximum velocity found in the velocity field $\left(u_{\max }\right)$ :

$$
u_{l}^{*}=\frac{u_{l}}{u_{\max }},
$$




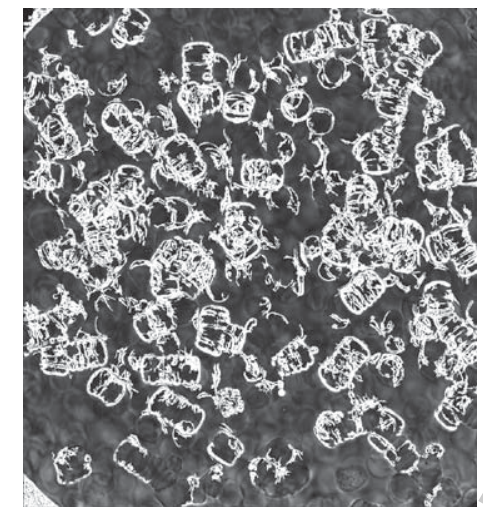

(a)

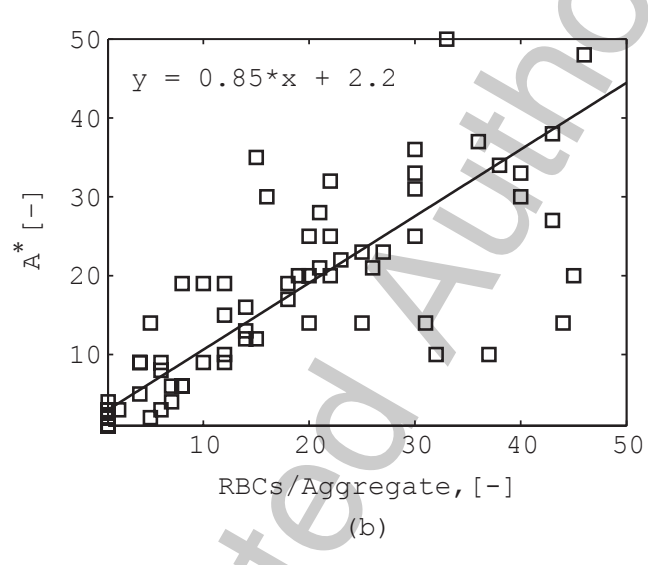

Fig. 6. Results of the third step of the validation process. (a) the detected edges when the image of Fig. 5(a) was blended with a high concentration dispersed RBC image (small areas are not shown for clarity). The correlation of $A^{*}$ to the actual number of RBCs per aggregate is shown in (b). The equation of fit shows that the slight underperformance is exaggerated, with a norm of fit at 3.4 for this case.

The general definition of the local shear rate magnitude for the present configuration was:

$$
|\dot{\gamma}|=\left[\left(\frac{\partial u^{*}}{\partial y^{*}}\right)^{2}+\left(\frac{\partial u^{*}}{\partial z *}\right)^{2}\right]^{1 / 2} \approx\left[\left(\frac{\Delta u^{*}}{\Delta y *}\right)^{2}+\left(\frac{\Delta u^{*}}{\Delta z *}\right)^{2}\right]^{1 / 2},
$$

\subsection{Hematocrit estimation}

The non-uniform and asymmetric concentration of RBCs in the cross stream direction (see Fig. 2) was quantified as a profile according to Sherwood et al. [51]:

$$
H\left(y^{*}\right)=\alpha\left(1-I\left(y^{*}\right)\right),
$$


where $I\left(y^{*}\right)$ is the intensity values in the $y^{*}$, cross-steam, direction (normalised by the width of the channel). In the present work, however, the calibration factor $\alpha$ was assumed to be equal to the ratio of the feed haematocrit $H_{f}$ to the mean intensity within the channel $\left(1-\bar{I}\left(y^{*}\right)\right)$ :

$$
\alpha=\frac{H_{f}}{\left.1-\bar{I}\left(y^{*}\right)\right)},
$$

i.e. the mean value of the intensity profile within the channel is equal to the feed haematocrit. The correction $\left(1-I\left(y^{*}\right)\right)$ is necessary due to the inverse relationship between the image intensity and haematocrit (higher intensity indicates lower hematocrit). It should be noted that the approximation of haematocrit as a linear function of intensity used here was considered appropriate since the feed hematocrit was relatively low (25\%). In general, the intensity is affected non-linearly by the haematocrit, depending on the optical properties of the system as well as the geometry of the flow configuration $[33,44]$.

\subsection{Statistical analysis}

The measured and calculated quantities (pixel intensity, velocity magnitude, edge area, etc.) are presented in following sections as spatial and/or temporal mean values where needed. Correlations between any two quantities A and B are evaluated using the correlation coefficient defined as:

$$
R=\frac{\operatorname{Covariance}(A, B)}{\sigma_{A} \sigma_{B}},
$$

with $\sigma$ being the standard deviation. The Pearson correlation coefficient ( $p$ value) is used for the level of significance in a correlation, and the significance of differences between the means of two not-normally distributed sets of data are assessed using the Wilcoxon Rank-Sum test as appropriate. Normality of data distributions were assessed with the one-sample Kolmogorov-Smirnov test, using Matlab (Mathworks) software.

The norm of fit, or sum of squared residuals (SSR), was used to quantify the goodness of the linear fitting in the correlations shown in Figs. 5 and 6. The general expression for SSR is:

$$
S S R=\sum_{i=1}^{n}(Y i-(a+b X i))^{2},
$$

where $X$ and $Y$ are two variables, $n$ is the number of points, and $a$ and $b$ are the $Y$ intercept and slop of the fitted line respectively.

\section{Results and discussion}

\subsection{Aggregation estimation using the contrast method}

Figure 7 shows the standard deviation of pixel value $\left(\sigma_{I}^{*}\right)$, which increases for the more highly aggregating cases (i.e. as $\dot{\gamma} \rightarrow 0$ ); this is in agreement with the fact that aggregation scales with the decrease of shear rate. No aggregation was detected above $\dot{\gamma} \approx 31.0 \mathrm{~s}^{-1}$. The moderately and highly aggregated states have distinctly elevated levels of $\sigma_{I}^{*}$ over baseline cases acquired at high pseudo shear rates and without Dextran 2000 in the suspending medium. In all aggregating cases, $\sigma_{I}^{*}$ reached a maximum within approximately 15s (Fig. 7(b)). 


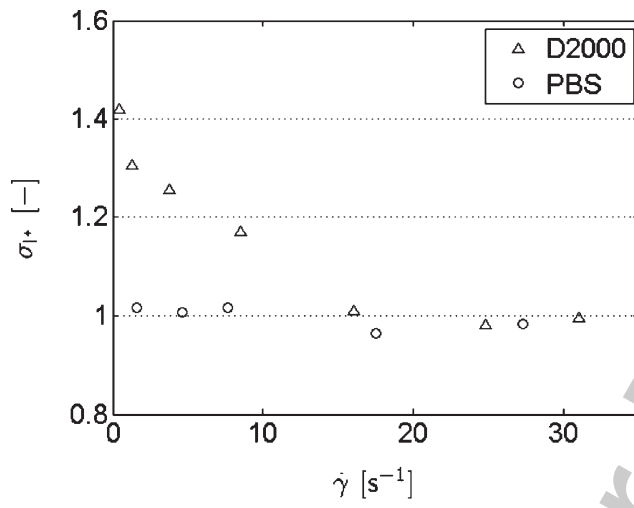

(a)

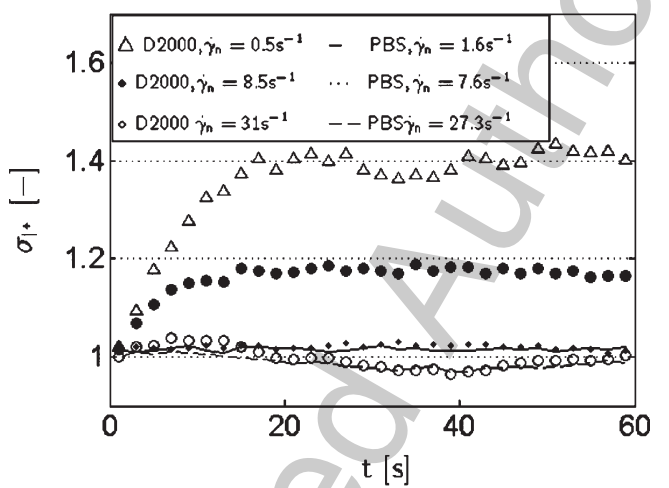

(b)

Fig. 7. Standard deviation of pixel intensity (contrast) (a) as a function of $\dot{\gamma}$; and (b) as a function of time for different pseudo shear rates. Cases with only PBS as the suspending medium are also shown on both plots as indicated. For comparison purposes, all values shown are normalised by the final value of the $\dot{\gamma}=31.0 \mathrm{~s}^{-1}$ case.

\subsection{Haematocrit gradients}

Mean hematocrit plots are shown in Fig. 8 for the non-aggregating (panel (a)) and aggregating cases (panel (b)). The profiles are ensemble averages of all instantaneous profiles in the last 10 seconds of the flow (49 to 59 seconds) from a high and a low pseudo-shear rate case (1.6 and $27 s^{-1}$ for the PBS case, and 1.3 and $25 s^{-1}$ for the D2000 case. In all cases slight hematocrit gradients with $y^{*}$ across the channel are present. Intensity peaks in the near-wall regions (see Fig. 2) are caused by syneresis, due to aggregation, and by axial migration of cells away from the walls; the heamatocrit at those regions is greatly reduced compared to the core of the flow. The standard deviation bars indicate that the maximum deviation in both cases is approximately $<5 \%$ heamatocrit, and that no significant differences are observed between the PBS and D2000 cases apart from the increased deviation observed for the low pseudo-shear rate $\left(1.3 s^{-1}\right)$ of the D2000 case. These temporal haematocrit fluctuations have been deemed responsible for perturbations in the velocity profile of even non-aggregating blood flows [32]; this issue is discussed in a following section. Figure 8 also illustrates that erythrocyte aggregation slightly exaggerates the variation in the local haematocrit. 


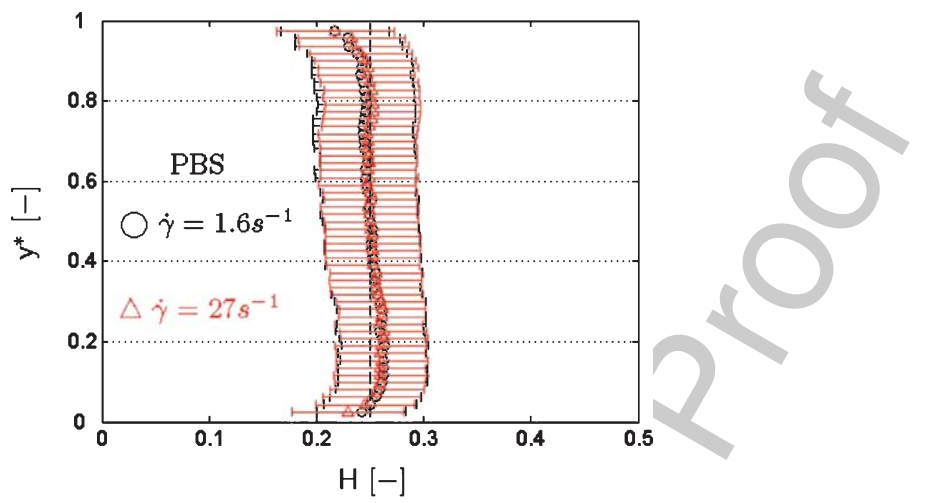

(a)

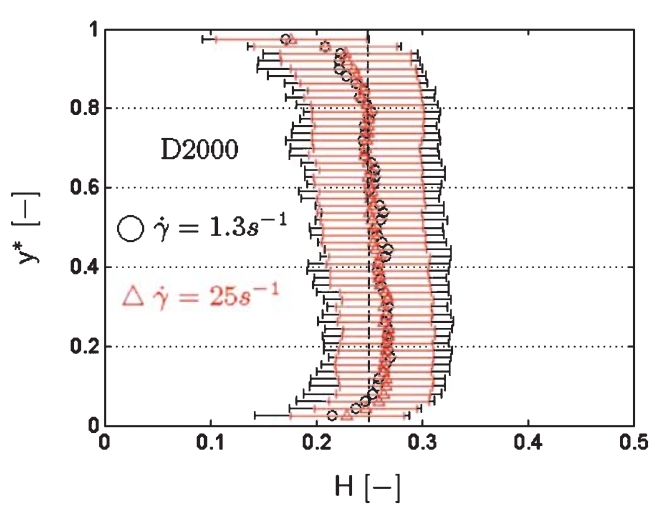

(b)

Fig. 8. Mean hematocrit profiles obtained by averaging the last 10 seconds of the images recorded. a) PBS, $1.6 s^{-1}$ (open circles) and $27 s^{-1}$ (open triangles). b) D2000, $1.3 s^{-1}$ (open circles) and $25 s^{-1}$ (open triangles). The standard deviation bars are included and the vertical dashed line indicates the feed hematocrit.

\subsection{Effect of aggregation and haematocrit gradients on velocity profiles}

Axial mean velocity profiles, resulted from the ensemble averaging of the instantaneous velocity fields for the last 10 seconds (according to [38]), are shown in Fig. 9(a); for both PBS and D2000 cases the measured velocity vectors were first spatially averaged in the $x$ direction for each image, then temporally averaged for the last 10 seconds of the flow (from the 49th to 59th second) and presented for two selected pseudo-shear rates (1.6 and $27 s^{-1}$ for the PBS case, and 1.3 and $25 s^{-1}$ for the D2000 case). The velocities were normalised by the maximum measured values for each case to facilitate easy comparison. The standard deviation indicated by the error bars illustrates that the differences between the velocity profiles (although not significant) become more pronounced for the low pseudo-shear rate and in the near wall regions of the channel.

Panel (b) in Fig. 9 shows the standard deviation of velocity calculated by first subtracting the mean profile from the instantaneous vector fields in the channel and analysing over the final $10 \mathrm{~s}$ of data acquired at each pseudo-shear rate for the D2000 and PBS cases. Figure 9(b) indicates that local deviations from the mean velocity fields occurred for the cases corresponding to a higher degree of erythrocyte aggregation, which is particularly noticeable for $\dot{\gamma}=1.3 \mathrm{~s}^{-1}$. These variations are not random noise, but occur over 


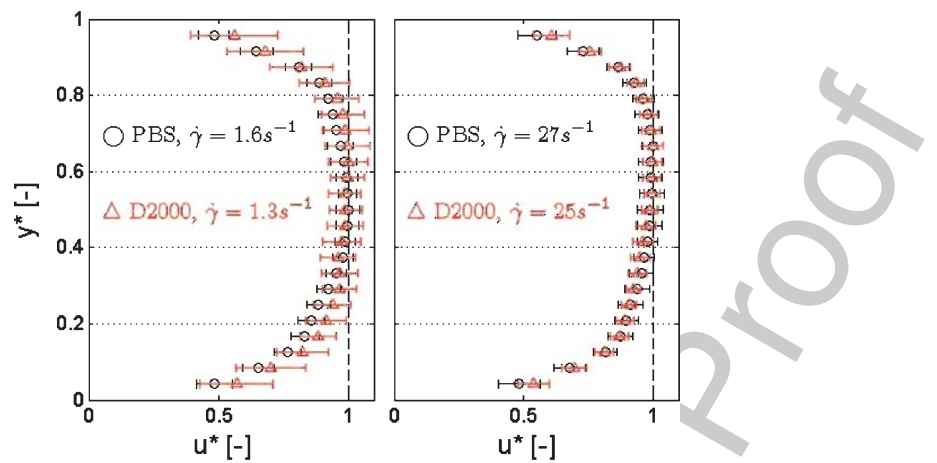

(a)

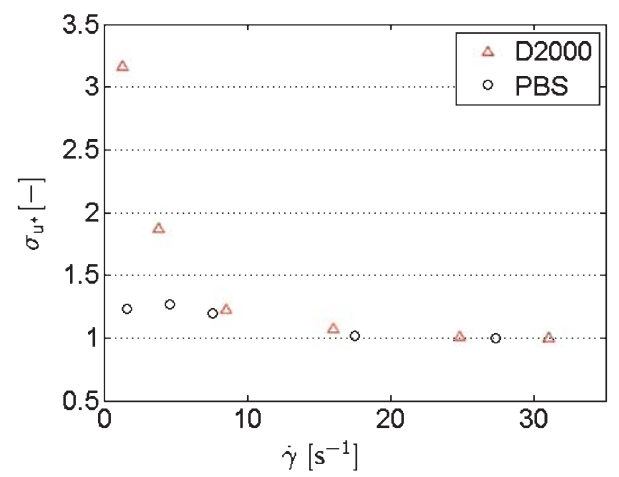

(b)

Fig. 9. (a): velocity profiles resulted from the ensemble averaging of the instantaneous velocity fields for the last 10 seconds of the flow (49th to 59th second), for a high and a low pseudo-shear rate; 1.6 and $27 s^{-1}$ for the PBS case, and 1.3 and $25 s^{-1}$ for the D2000 case. $u^{*}$ is the velocity normalised by the maximum velocity occurring in each case. (b): standard deviation of the normalised velocities (expressed as a ratio to the mean value) as a function of pseudo shear rate for the D2000 and PBS cases.

several spatially and/or temporally adjacent vectors, and are present in both the bulk flow and near wall regions of the microchannel. These localised and temporary decreases in velocity can be attributed to larger aggregates resisting the flow more and interacting with the wall. In contrast, the non-aggregating control samples exhibited instantaneous vector fields that were closer to the mean channel flow and changes with pseudo-shear rate were not observed. The velocity profiles in Fig. 9 were derived from fully developed flows, and obtained from the last 10 seconds of a 59-second flow duration to ensure steadiness. Therefore it is expected that the aggregate size evolution would have reached equilibrium at this conditions.

Figure 9 reveals two important characteristics regarding the influence of haematocrit gradients and RBC aggregation on blood flow; firstly, the haematocrit gradients affect the velocity profile by skewing it towards the lower RBC concentrations and secondly that aggregation increases both the bluntness of the velocity profile and the local velocity variation. These observations agree with our previously reported findings [50, 51].

\subsection{Microstructural characteristics of blood derived by the edge-detection method}

Figure 10 illustrates the performance of the edge detection algorithm on two representative cases; sections of processed images from a PBS case $\left(\dot{\gamma}=1.6 s^{-1}\right)$ and from a D2000 case $\left(\dot{\gamma}=1.3 s^{-1}\right)$ are 


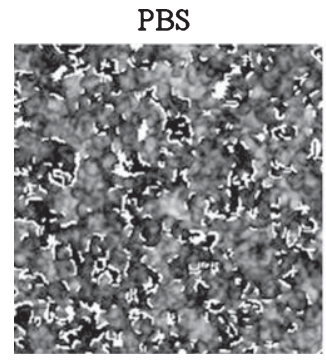

(a)

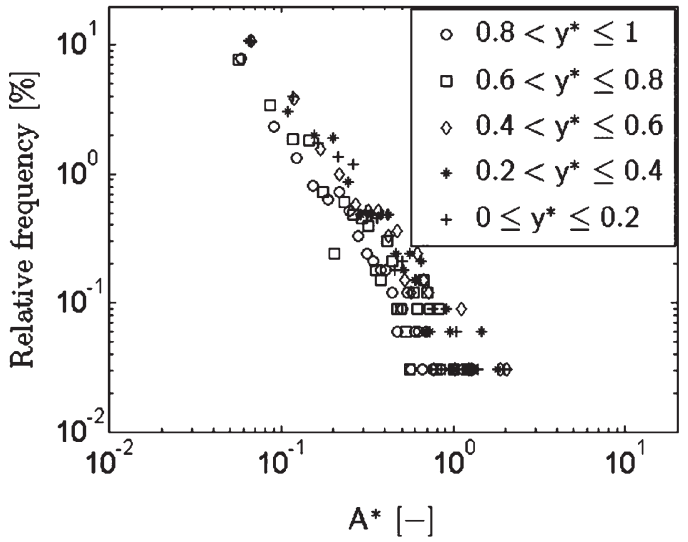

(c)

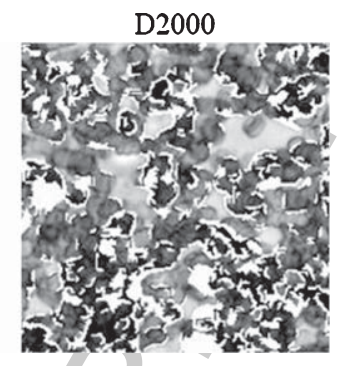

(b)

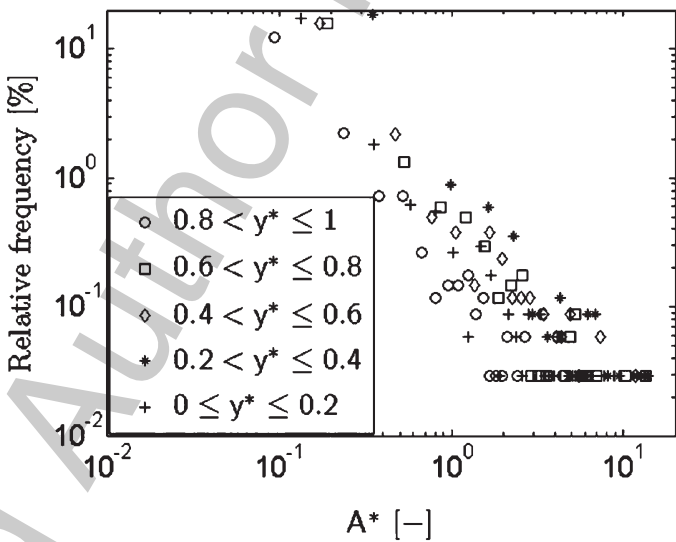

(d)

Fig. 10. Top panel: Selected edge detection images applied to (a) PBS case $\left(\dot{\gamma}=1.6 \mathrm{~s}^{-1}\right)$ at $t=59 \mathrm{~s}$, and (b) D2000 case $\left(\dot{\gamma}=1.3 \mathrm{~s}^{-1}\right)$ at $t=59 \mathrm{~s}$. Bottom panel: Area distributions of detected structures for the PBS (c) and D2000 (d) cases within the following regions of the channel; $0 \leq y^{*} \leq 0.2(+), 0.2<y^{*} \leq 0.4\left(^{*}\right), 0.4<y^{*} \leq 0.6 \sim(\diamond), 0.6<y^{*} \leq 0.8 \sim(\square)$ and $0.8<y^{*} \leq 1 \sim(\circ)$; (c) PBS case and (c) D2000 case (d) $A^{*}$ is the structure size normalised by the edge size of one RBC

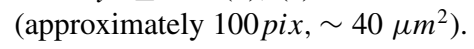

shown in panels (a) and (b) respectively for $t=59 \mathrm{~s}$. The structures revealed by the edge-detection algorithm show distinct differences in terms of size and shape. The differences in size were analysed quantitatively and presented in Fig. 10(c) and (d), where the size distribution of the detected structures in the entire images corresponding to the image sections presented in Figs. 10(a) and (b) are plotted in a log$\log$ plot. $A^{*}$ is a dimensionless quantity representing detected structure area normalised by the edge area of an imaged RBC (approximately 100 pixels or $40 \mu \mathrm{m}^{2}$ ), hence $A^{*}=1$ corresponds to the area of one $\mathrm{RBC}$. The frequency of occurrence is presented relative to the total number of structures in the image. The size distributions shown in Fig. 10(c) and (d) have been obtained from different regions (bands) within the channel and represented with different symbols. The channel was divided into five bands in the $y$ direction, as shown in Fig. 9(a), in order to isolate the regions of high velocity and hematocrit gradients, and the following limits were set: $0 \leq y^{*} \leq 0.2,0.2<y^{*} \leq 0.4,0.4<y^{*} \leq 0.6,0.6<y^{*} \leq 0.8$ and $0.8<y^{*} \leq 1$.

The distribution of the non-aggregating case in Fig. 10(c) shows no large variation between the different bands as expected and no structures larger than one $\operatorname{RBC}\left(A^{*} \approx 1\right)$ are present in the channel. In the aggregating case of Fig. 10(d) the size of the detected structures increases to several times the RBC size and the spread between the cases is greater than the one observed in the non-aggregating case. Although 


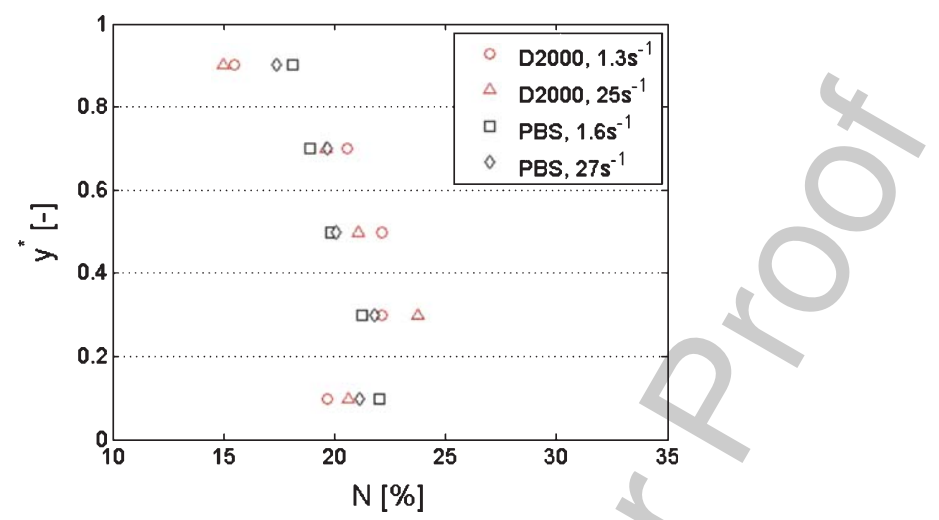

Fig. 11. Comparison of the number of detected edges in each band for the PBS and D2000 cases, for two different pseudo-shear rates (1.6 and $27 s^{-1}$ for the PBS case, and 1.3 and $25 s^{-1}$ for the D2000 case) and for the 59th second of the flow.

not clearly visible Fig. 10(d) shows significant differences ( $p \ll 0.05$, Wilcoxon rank sum test) between the central band and those near the wall of the channel when considering large structures $\left(A^{*}>1\right)$; it should be noted that areas smaller than $A^{*}=1$ may indicate both a single cell and part of an aggregate, but an $A^{*}>3$ clearly indicates an aggregate.

The number of structures detected within each band $(N)$, expressed as a percentage of the total number of structures detected in the whole image, is shown in Fig. 11 (for two selected psedo-shear rates and for the 59th second of the flow) from a representative image. The trends revealed in this figure agree qualitatively with those observed in Fig. 8 although $N$ has not been calibrated for haematocrit. It is also noted that the differences between PBS and D2000 shown in Fig. 11 are stronger within the $0.8<y^{*} \leq 1$ band. The agreement of the results produced by the edge-detection technique with those obtained by the bulk indices $\sigma_{I^{*}}$ (Fig. 7(a)) and $\sigma_{u^{*}}$ (Fig. 9(b)) is illustrated in Fig. 12(a). In this figure the mean structure area $\left(\bar{A}^{*}\right)$, averaged over the whole image and for the last 10 seconds of the flow, is shown as a function of the pseudo-shear for the D2000 and PBS cases. Figure 12(b) shows the mean structure area as a function of time. The behaviour of $\bar{A}^{*}$ follows closely that of $\sigma_{I^{*}}$ seen in Fig. 7(b).

The influence of the shear conditions on the development of the structures at different regions of the channel is illustrated in Fig. 13. The mean edge area $\left(\bar{A}^{*}\right)$ in each band, is shown for low pseudo-shear rates and for the 59th second of the flow for the D2000 and PBS cases. No trends are observed for the PBS case apart from a slight increase of the mean size for the bands where the haematocrit was higher (i.e. towards the $0 \leq y^{*} \leq 0.2$ band) indicating perhaps a limitation of the technique. In contrast the results from the aggregating case show that the technique captures very well the known influence of both the time and shear conditions on the development of aggregates [23]. It should be noted here that in the first few seconds of the flow the values of $\bar{A}^{*}$ for the D2000 case are very close to 1 , indicating negligible aggregation.

\subsection{Velocity characteristics of detected structures}

The sensitivity of the method for capturing more details of the relationship between structural characteristics and flow was further assessed and illustrated in Fig. 14; the area of the detected structures was studied against the corresponding local mean velocity $u_{l}^{*}$ (results presented for 50 processed images from the last 10 seconds of $\dot{\gamma}=4.6$ and $\dot{\gamma}=3.8 s^{-1}$ for the PBS and D2000 cases respectively). No 


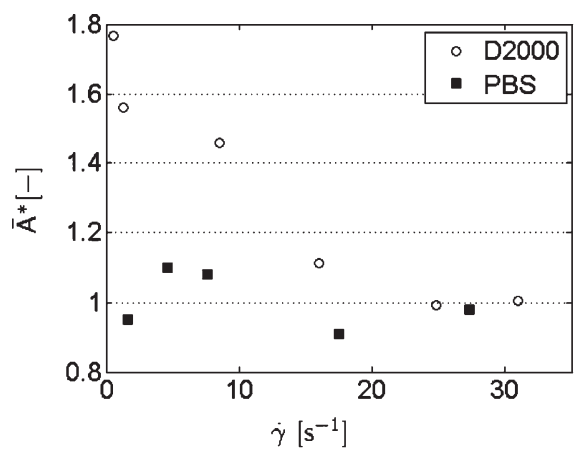

(a)

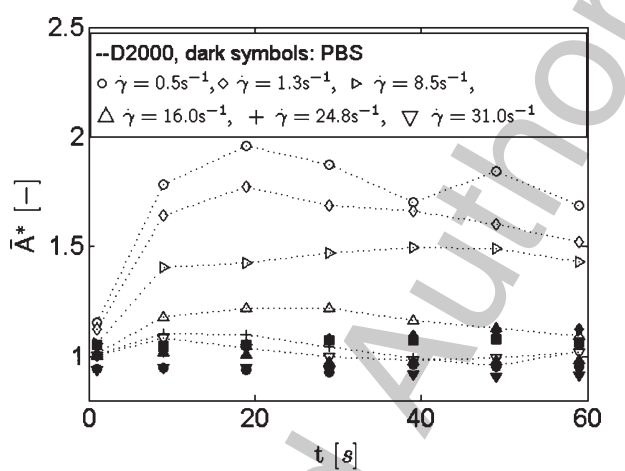

(b)

Fig. 12. (a): comparison of the mean area of detected edges in the channel for the PBS and D2000 cases (averaged for the whole image and for the last 10 seconds of the flow). (b): mean structure area in the channel averaged at each time point of measurement (100 images) for the D2000 (dashed lines and open symbols) and PBS (dark symbols) cases.

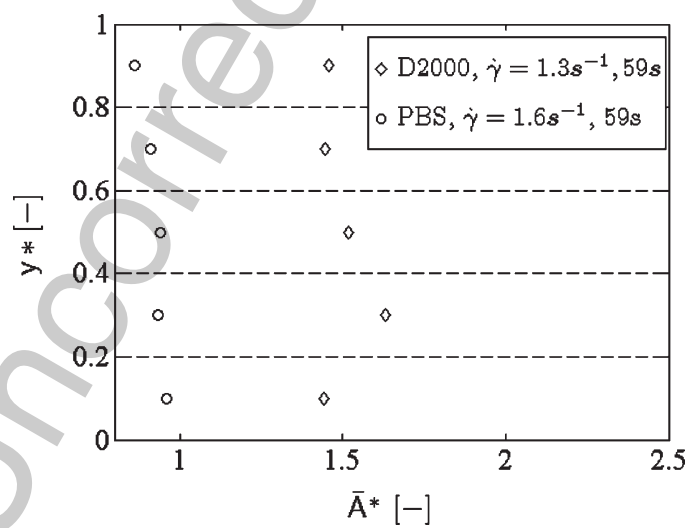

Fig. 13. Mean edge area detected in each band for low pseudo-shear rate cases (1.3, and $1.6 s^{-1}$ for the D2000 and PBS cases respectively) and for the 59th second of the flow.

trends are observed for the PBS case in Fig. 14(a), apart from those in the velocity profile of Fig. 9 (i.e. maximum velocity occurring within the $0.4 \leq y^{*} \leq 0.8$ region) and a narrowing of the velocity variation with the increase of $A^{*}$ (probably resulting from the local averaging of the velocity vectors). The D2000 


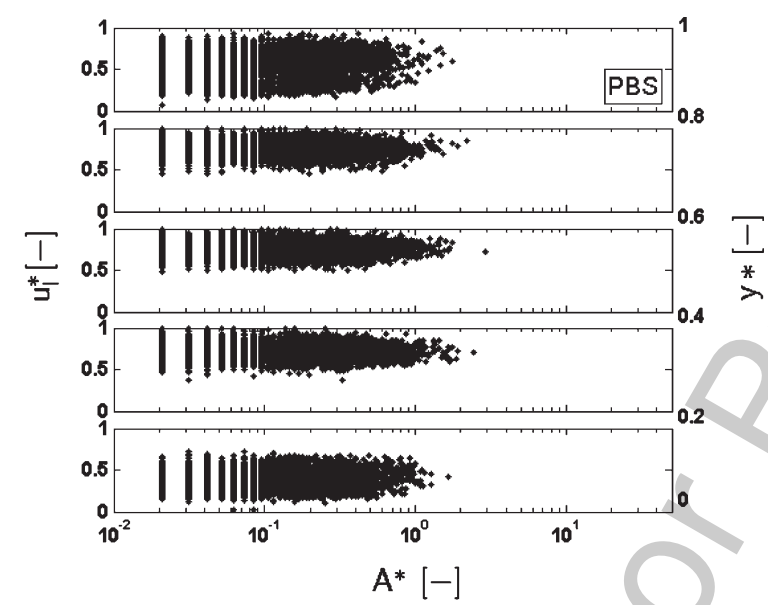

(a)

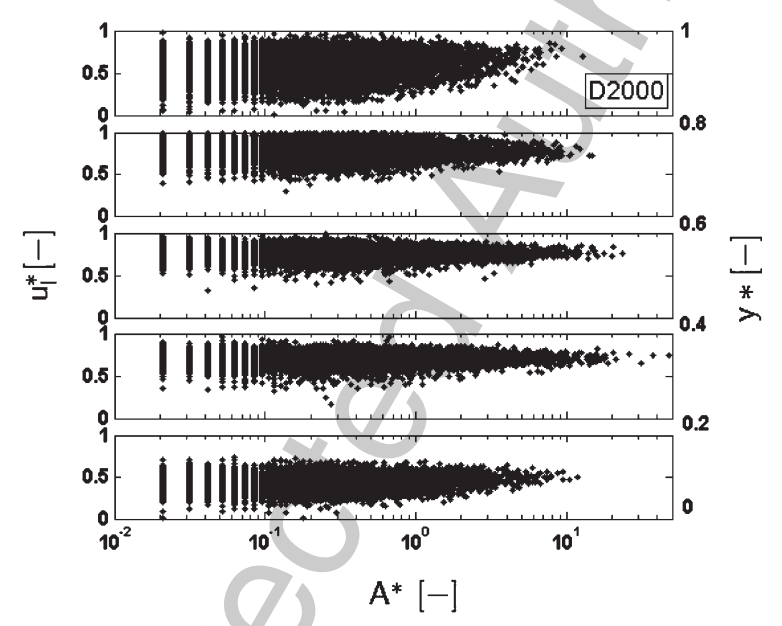

(b)

Fig. 14. Mean local velocity $\left(u_{l}^{*}\right)$ against structure size $A^{*}$ for each flow band ( $y^{*}$ band limits are shown in the right vertical axis). The upper panel shows a PBS case $\left(\dot{\gamma}=4.6 s^{-1}\right)$ and the bottom panel the D2000 case $\left(\dot{\gamma}=3.8 s^{-1}\right)$. The results are presented for 50 processed images from the last 10 seconds of both cases).

case (panel (b)) clearly shows that the larger structures tend to be associated with the highest velocities for $A^{*}>1$ within the two extreme bands. Quantitatively, a positive correlation coefficient $(\mathrm{R})$ of $\approx 0.5$ ( $\mathrm{p}<<0.001$ ) is observed when correlating $u_{l}^{*}$ with $A^{*}$ within the $0 \leq y^{*} \leq 0.2$ and $0.8 \leq y^{*} \leq 1$ bands (for $A^{*}>1, \mathrm{p}<<0.001$ and $\mathrm{R} \approx 0.2$ in both extreme bands). This might be due to fact that for the high aspect ratio geometry employed in the present study the highest velocities within the extreme bands may correspond to sub-regions of smaller velocity gradients (see the velocity profiles in Fig. 9).

\subsubsection{A note on the spatiotemporal variations of hematocrit and velocity}

It was mentioned in Section 3.3 that the local spatial and temporal variation in velocity may be due to aggregates, interacting with the walls and resisting the flow. It was also observed that the nonaggregating control samples showed smaller, but finite variations in the velocity magnitude. Figure 8 
illustrates that hematocrit variations are present in both aggregating and non-aggregating cases. Similar variations in velocity and hematocrit, for constant discharge hematocrits and no axial variation in vessel diameters, have been reported in the literature and attributed to hemodynamic factors alone [26]. In the study of [26] the effect of hematocrit variation on the velocity magnitude was analysed and it was found that it has an influence on the variation of flow parameters; this is confirmed in the present study also (see the hematocrit and velocity variation in Figs. 8 and 9). In addition, Fig. 14 further clarifies the fact that another possible factor contributing in the spatiotemporal variation of velocity is the aggregation phenomenon, which results in altering the microstructure of blood. As shown in the previous section large aggregates tend to be associated with the larger velocities, however, Fig. 14b shows that even in the central regions of the channel a distribution of velocities corresponding to specific $A^{*}$ values larger than unity exists, indicating the variability in the velocity magnitude. The effect of shear on hematocrit and velocity variation is observed to be negligible for the non-aggregating, but pronounced for the aggregating cases (Figs. 8 and 9b). Therefore it can be concluded that the shear conditions influence the variability in hematocrit and velocity through the shear-dependent phenomenon of RBC aggregation. Nevertheless, the causes of hematocrit variation are unclear. For the aggregating cases it could be argued that the non-uniform distribution of aggregate size and their complex interactions could have an effect on the variation of hematocrit (see for example [22]). For the nonaggregating cases, the deformability of the RBCs may be the major factor influencing the hematocrit variation.

\subsection{Shear effects on structure size near the side-wall of the channel}

To clarify the observations made in the previous section $A^{*}$ was correlated with the local shear rate magnitude $|\dot{\gamma}|$ for the $0 \leq y^{*} \leq 0.1$ region. This region was chosen because the $\frac{\partial u^{*}}{\partial y^{*}}$ component of the local shear rate was expected to dominate over the $\frac{\partial u^{*}}{\partial z^{*}}$ component and therefore the velocity profile could suffice for the estimation of the shear rate in that particular region. For most part of the high aspect ratio microchannel, the shear rate is significantly affected by the velocity profile in the $z$ direction, and in the absence of the latter the calculation of the actual mean shear rate is not possible. The marked skewness of the velocity profile towards the $0.6 \leq y^{*} \leq 0.8$ region of the channel (see Fig. 9(a)) is expected to further contribute to the domination of the $\frac{\partial u^{*}}{\partial y^{*}}$ component in the $0 \leq y^{*} \leq 0.1$ region. For the reasons explained above and in view of the absence of the $\frac{\Delta u^{*}}{\Delta z^{*}}$ component of shear, it was deemed appropriate to investigate the influence of only the in-plane shear rate component on the development of aggregates in the near wall region $0 \leq y^{*} \leq 0.1$, i.e.:

$$
|\dot{\gamma}|=\left[\left(\frac{\Delta u^{*}}{\Delta y *}\right)^{2}\right]^{1 / 2} .
$$

The shear rate magnitude was normalised by the smallest calculated value found in the region, in order to present the data as multiples of the lowest shear rate occurring in the region:

$$
\left|\dot{\gamma}^{*}\right|=\frac{|\dot{\gamma}|}{\left|\dot{\gamma}_{\min }\right|}
$$

The results presented in Fig. 15(a) show $A^{*}$ against $\left|\gamma^{*}\right|$, for the $0 \leq y^{*} \leq 0.1$ region and for 50 images captured within the last 10 seconds of the D2000 case at a pseudo-shear of $3.8 \mathrm{~s}^{-1}$. Figure 15(b) shows the data only for $A^{*}>1$ and illustrates that RBC aggregation occurs in regions of low shear rate; this is 


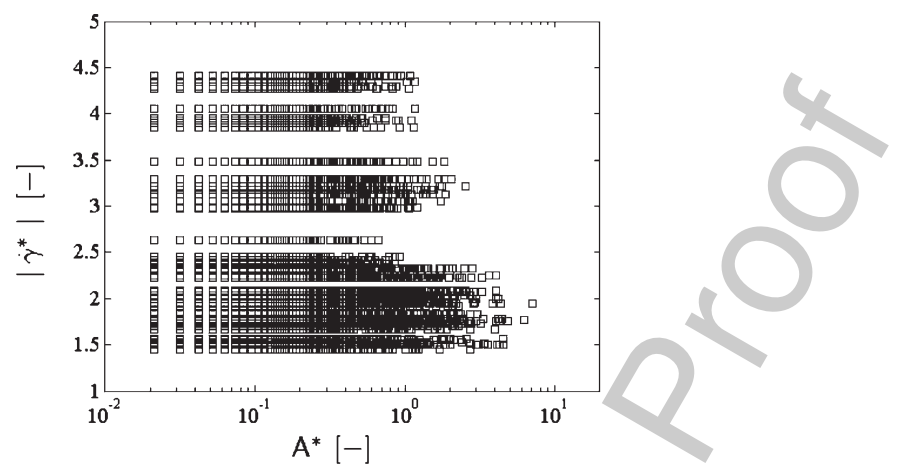

(a)

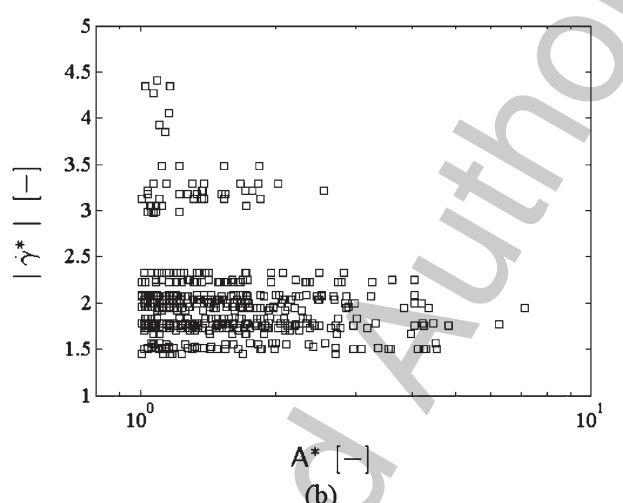

(b)

Fig. 15. (a): Local shear rate magnitude $\left(\left|\gamma^{*}\right|\right)$ against structure size for the D2000 $\left(\dot{\gamma}^{*}=3.8 s^{-1}\right)$ case. (b) shows the data for $A^{*}>1$; the correlation coefficient found to be $-0.2(p<<0.001)$.

well documented in the literature (see for example [23]). A moderate negative correlation of $A^{*}$ with $\left|\gamma^{*}\right|$ is observed for the data in Fig. 15(a) $(\mathrm{R}=-0.4, p<<0.001)$, although this correlation becomes weaker when considering $A^{*}$ above 1 for the data in Fig. 15(b) $(\mathrm{R}=-0.2, p<<0.001)$.

\subsection{Shear effects on structure size away from the side-walls of the channel}

Figures 14(b) and 13 illustrate that the larger structures found in the channel develop in the $0.2 \leq y^{*}$ $\leq 0.6$ region, indicating that this may be the region of the lowest actual mean shear rate in the channel. Considering the velocity and structure size characteristics within the $0.4<y^{*} \leq 0.6$ and $0.6<y^{*} \leq 0.8$ regions it is observed that although the mean velocity is similar (see Fig. 9(a)), the area of the detected structures is slightly smaller within the $0.6<y^{*} \leq 0.8$ band (see Figs. 13 and 14(b)). This may be explained by the small erythrocyte concentration in the $0.6<y^{*} \leq 0.8$ region (see Fig. 8(b)) and from the fact that the $\frac{\partial u^{*}}{\partial z *}$ component of the shear rate is expected to influence and increase the actual mean shear rate in this region. It is also observed from Fig. 14(b) that the maximum detected area is found within the $0.2<y^{*} \leq 0.4$ band, where the maximum erythrocyte concentration (see Fig. 8(b)) and the minimum velocity within the bulk flow occur (see Fig. 9(a)), indicating a combined effect of the shear conditions and erythrocyte concentration. 


\section{Limitations of the image processing methods}

One of the most important factors that influence the performance of both image processing methods and the $\mu$ PIV technique is the depth of focus due to the microscopy system set up. The current system with a 10x magnification objective and a 0.25 numerical aperture resulted in a depth of focus that engulfs the entire depth of the channel. The nature of the fluid, which is a dense suspension of biconcave RBCs and, at low shearing flows, aggregates, further contributes to the large depth of correlation. The limitations of the $\mu$ PIV technique when PIV algorithms are carried out on images of RBCs have been discussed in detail in Sherwood et al. [51]. The large depth of focus also did not allow the measurement of velocities at different locations in the $\mathrm{z}$ direction and therefore the $\frac{\partial u^{*}}{\partial z^{*}}$ component of shear, which dominates in the largest proportion of the bulk flow, could not be evaluated. The absence of the out-of-plane shear rate affected the estimation of the effective shear within the channel and restricted the analysis of the local shear in relation to the aggregate size for a small portion of the channel $\left(0 \leq y^{*} \leq 0.1\right)$, where the in-plane component of shear $\left(\frac{\partial u^{*}}{\partial y^{*}}\right)$ was deemed dominant over the $\frac{\partial u^{*}}{\partial z^{*}}$ component. More powerful lenses, in combination with image pre-processing, could help in order to reduce the depth of field and the depth of correlation in the system.

As mentioned previously, the large depth of focus and the multiple layers of cells within the channel cause the single-RBC detected edges to overlap resulting in edge areas not corresponding to single RBCs. To rectify this the gradient threshold values were kept at levels whereby this phenomenon was minimised, so that the mean detected edge area to correspond to the edge area of one RBC. The disadvantage of the aforementioned approach was that the density of the detected structures was obviously lower than that of the embedded RBCs (see Fig. 2a). This raises a question on the spatial resolution that the statistical analysis of the results is valid in relation to a) the calculation of the relative number of structures (i.e. haematocrit) and b) the identification of the large structures, given the fact that aggregates are several times larger than the RBCs (see Fig. 2b). The minimum spatial resolution used in the present work was set to $50 \mu \mathrm{m}$, i.e. the width of the bands dividing the channel, according to the analysis made in $\mathrm{Xu}$ et al. [61]; in that study the spatial correlation of image gray-scales at low shear rates showed that $\approx 7$ to $8 \mathrm{RBC}$ diameters are sufficient to cover the large aggregate areas.

The slight bias of the edge detection method towards the higher concentrations of cells is seen in Fig. 13, where the detected size of the structures in the PBS case seems to increase in regions of higher haematocrit. However, the significance of this bias seems small when comparing the PBS to the D2000 case in Fig. 13. Another consequence of setting the gradient threshold high is seen in Fig. 10(b), which illustrates that the shape of the aggregates has not been completely recovered by the edge-detection method, however, large parts of their distinctive configuration were revealed. It should be noted that in the present work image pre- and post-processing operations (morphological filtering, etc.) were kept to a minimum in order to preserve the details of the structure appearing in the micro-scale.

Although the image processing methods developed in the present work were found to be powerful techniques for empirically characterising $\mathrm{RBC}$ aggregation, further refinement would be required to allow better spatial resolution in order to investigate specific regions of interest in a variety of micro-scale flows (e.g. bifurcations and networks). Hybrids of the powerful Canny edge-detection method could be developed to analyse the varying intensity gradients captured in the images, and analysis in the frequency domain (spectral measures of texture, etc.) could be employed to characterise ordered structures, such as rouleaux [17]. 


\section{Conclusions}

The manner in which erythrocyte aggregation affects flow behaviour, thereby influencing the broader rheological properties of blood, is an area of haemodynamics which has significant knowledge gaps, despite its relevance to physiological and pathological conditions. In the current study, microscopy imaging techniques have been used to simultaneously quantify the state of cell aggregation within a blood sample flowing through a microchannel, as well as the corresponding velocity fields and haematocrit profiles. These velocity profiles can then be used to determine other properties such as flowrate, pseudo-shear rate and the in-plane shear distribution. For higher pseudo-shear rates, in which cells are disaggregated by hydrodynamic stresses, the variation in the time-resolved velocity profiles is small. As the pseudo-shear rate decreases, the cells begin to aggregate, which increasingly affects the instantaneous velocity fields and the variation observed. Local spatial and temporal deviations from the expected velocity profiles increase with aggregation, which is primarily due to the interaction of erythrocyte aggregates with each other and the channel walls. In addition, the aggregation of RBCs was found to affect the bluntness of the velocity profile, compared to the non-aggregating case, and the haematocrit gradients to influence the skewness of the velocity profiles in agreement with our previous studies [50,51]. The image contrast and velocity variance both provide quantitative indications of the presence of aggregation at different levels, and could be further developed for research and medical purposes. The edge detection method developed in the present work showed that it is possible to probe the structural properties of blood at a local level; more specifically, within the side wall regions of the channel the larger structures were found to develop in sub-regions of higher velocities and lower shear magnitude. Within the bulk flow (i.e. away from the side-walls) the largest structures occur in regions of high erythrocyte concentrations and lower velocities. The current study illustrates that it may be possible to combine different methods for quantifying aggregation, for a more robust characterisation of the microstructure of blood. The various limitations imposed by the nature of the fluid and the high aspect geometry, require a further refinement of the image processing techniques in order to fully explore their potential. Nevertheless, the study demonstrates that the local microstructural characteristics of blood can be captured and analysed in relation to the local flow characteristics by using the proposed methods. An important next step will be to use this approach for simultaneous measurement of erythrocyte aggregation, distribution and velocity fields in more complex geometries involving bifurcations and more heterogeneous velocity and hematocrit distributions.

\section{Conflict of interest statement}

No conflict of interest to declare.

\section{Acknowledgements}

The authors would like to thank Dr Peter Vennemann for providing his PIV processing software online (www.jpiv.vennemann-online.de), as well as Emeritus Prof Roy Pike, Physics, Kings College London, for providing some equipment and Dr. D. Chase and Ms D. Johnson from King's Health Centre for their assistance. This work was supported in part by the EPSRC Life Sciences Interface program (EP/F007736/1). 


\section{References}

[1] S. Arscott, SU-8 as a material for lab-on-a-chip-based mass spectrometry, Lab on a Chip 19 (2014), 3668-3689.

[2] G. Barabino, M.O. Platt and D. Kaul, Sickle cell biomechanics, Annual Review of Biomedical Engineering 12 (2010), 345-367.

[3] J. Barbee and G. Cokelet, The Fåhraeus effect, Microvascular Research 3 (1971), 6-16.

[4] J. Bishop, P. Nance, A. Popel, M. Intaglietta and P. Johnson, Effect of erythrocyte aggregation on velocity profiles in venules. American Journal of Physiology: Heart and Circulatory Physiology 280 (2001), H222-H236.

[5] J. Bishop, A. Popel, M. Intaglietta and P. Johnson, Effect of aggregation and shear rate on the dispersion of red blood cells flowing in venules, American Journal of Physiology: Heart and Circulatory Physiology 283 (2002), H1985-H1996.

[6] A.W. Browne, L. Ramasamy, T.P. Cripe and C.H. Ahn, A lab-on-a-chip for rapid blood separation and quantification of haematocrit and serum analytes, Lab on a chip 11 (2009), 2440-2446.

[7] H. Bruus, 2008. Theoretical Microfluidics, Oxford University Press, Oxford, UK.

[8] P. Carpentier, B. Imbert, C. Picart and C. Maltere, Morphometric analysis of erythrocyte aggregates in vitro: pharmacological application with buflomedil, Journal des Maladies Vasculaires 25(5) (2000), 356-359.

[9] S. Chien and K. M. Jan, Ultrastructural Basis of the Mechanism of Rouleaux Formation, Microvascular Research 5 (1973), 155-166.

[10] S. Chen, B. Gavish, Y. Mahler and S. Yedgar, Monitoring of erythrocyte aggregate morphology under flow by computerised image analysis, Biorheology 32(4) (1995), 487-496.

[11] G. Cokelet and H. Goldsmith, Decreased hydrodynamic resistance in the two-phase flow of blood through small vertical tubes at low flow rates, Circulation Research 68 (1991), 1-17.

[12] E. Damiano, D. Long and M. Smith, Estimation of viscosity profiles using velocimetry data from parallel flows of linearly viscous fluids: application to microvascular haemodynamics, Journal of Fluid Mechanics 512 (2004), 1-19.

[13] J. Dusting, E. Kaliviotis, S. Balabani, and M. Yianneskis, Coupled human erythrocyte velocity field and aggregation measurements at physiological haematocrit levels, Journal of Biomechanics 42 (2009), 1438-1443.

[14] Y. Fung, 1997, Biomechanics: Circulation. 2nd edition. Springer.

[15] P. Gaehtgens, F. Kreutz and K. Albrecht, Fåhraeus effect and cell screening during tube flow of human blood. ii. effect of dextran-induced cell aggregation, Biorheology 15 (1978), 155-161.

[16] H. Goldsmith and J. Marlow, Flow behavior of erythrocytes. ii, Particle motions in concentrated suspensions of ghost cells, Journal of Colloid and Interface Science 71 (1979), 383-407.

[17] R. C. Gonzales, R.E. Woods and S. L. Eddins, 2004, Digital image processing, using Matlab. Prentice Hall.

[18] J.V. Green, T. Kniazeva, M. Abedi, D.S. Sokhey, M.E. Taslim and S.K. Murthy, Effect of channel geometry on cell adhesion in microfluidic devices, Lab on a chip $\mathbf{9}$ (2009), 677-685.

[19] P. Gyawali, R.S. Richards, D.L. Hughes and P. Tinley, Erythrocyte aggregation and metabolic syndrome, Clinical Hemorheology and Microcirculation 57 (2014), 73-83.

[20] H. Ji and S. Lee, In vitro hemorheological study on the effect of human blood flow in a microtube, Clinical Hemorheology and Microcirculation 40 (2008), 19-30.

[21] F. Jung, C. Mrowietz, B. Hiebl, R.P. Franke, G. Pindur and R. Sternitzky, Influence of rheological parameters on the velocity of erythrocytes passing nailfold capillaries in humans, Clinical Hemorheology and Microcirculation 48 (2011), 129-139.

[22] E. Kaliviotis, J. Dusting and S. Balabani, Spatial variation of blood viscosity: Modelling using shear fields measured by a $\mu$ PIV based technique, Medical Engineering and Physics 33 (2011), 824-841.

[23] E. Kaliviotis and M. Yianneskis, Fast response characteristics of red blood cell aggregation, Biorheology, 45 (2008b), 639-649.

[24] E. Kaliviotis, I. Ivanov, N. Antonova and M. Yianneskis, Erythrocyte aggregation at non-steady flow conditions: A comparison of characteristics measured with electrorheology and image analysis, Clinical Hemorheology and Microcirculation 44 (2010), 43-54.

[25] A. Kesler, Y. Yatziv, I. Shapira, S. Berliner and E.B. Assayag, Increased red blood cell aggregation in patients with idiopathic intracranial hypertension. A hitherto unexplored pathophysiological pathway, Thrombosis and Haemostasis 96(4) (2006), 483-487.

[26] M.F. Kiani, A.R. Pries, L.L. Hsu, I.H. Sarelius, and G.R. Cokelet, Fluctuations in microvascular blood flow parameters caused by hemodynamic mechanisms, American Journal of Physiology - Heart and Circulatory Physiology 35 (1994), H1822-H1828. 
[27] A.G. Koutsiaris, D.S. Mathioulakis and S. Tsangaris, Microscope PIV for velocity-field measurement of particle suspensions flowing inside glass capilaries, Meas Sci Technol 10 (1999), 1037-1046.

[28] A.G. Koutsiaris, Digital Micro PIV ( $\mu$ PIV) and Velocity Profiles In Vitro and In Vivo, Ch. 11 in The Particle Image Velocimetry - Characteristics, Limits and Possible Appications, INTECH (2012), ISBN 978-953-51065-8.

[29] A. Kvon, Y.H Lee, T.A. Cheema, and C.W. Park, Development of dual micro-PIV system for simultaneous velocity measurements: optical arrangement techniques and application to blood flow measurements, Measurement Science and Technology 25 (2014), 075302. doi:10.1088/0957-0233/25/7/075302.

[30] C. Lehmann, I. Abdo, H. Kern, L. Maddison, D. Pavlovic, N. Sharawi, J. Starkopf, R. Hall, P. Johnson, L. Williams, V. Cerny, Clinical evaluation of the intestinal microcirculation using sidestream dark field imaging - Recommendations of a round table meeting, Clinical Hemorheology and Microcirculation 57(2) (2014), 137-146.

[31] R. Lima, S. Wada, M. Takeda, K. Tsubota and T. Yamaguchi, In vitro confocal micro-PIV measurements of blood flow in a square microchannel: The effect of the haematocrit on instantaneous velocity profiles, Journal of Biomechanics $\mathbf{4 0}$ (2007), 2752-2757.

[32] R. Lima, S. Wada, M. Takeda, K. Tsubota and T. Yamaguchi, In vitro blood flow in a rectangular PDMS microchannel:experimental observations using a confocal micro-PIV system, Biomedical Microdevices 10 (2007), 153-167.

[33] H. Lipowsky, S. Usami and S. Chien, In vivo measurements of apparent viscosity and microvessel hematocrit in the mesentery of the cat, Microvascular Research 19 (1980), 297-319.

[34] H. Lipowsky, Microvascular rheology and hemodynamics, Microcirculation 12 (2005), 5-15.

[35] H. Lipowsky, S. Kovalcheck and B. Zweifach, The distribution of blood rheological parameters in the microvasculature of cat mesentery, Circulation Research 43 (1978), 738-749.

[36] G. Mchedlishvili, N. Beritashvili, D. Lominandze and B. Tsinamdzvrishvili, Technique for direct and quantitative evaluation of erythrocyte aggregability in blood samples, Biorheology 30 (1993), 153-161.

[37] C.D. Meinhart, S.T. Wereley, and J.G. Santiago, PIV measurements of a microchannel flow, Experiments in Fluids 27 (1999), 414-419.

[38] C.D. Meinhart, S.T. Wereley, and J.G. Santiago, A PIV algorithm for estimating time-averaged velocity fields, Journal of Fluids Engineering 122 (2000), 285-289.

[39] H. Meiselman, Red blood cell aggregation: 45 years being curious, Biorheology 46 (2009), 1-19.

[40] M.G. Olsen and R.J. Out-of-focus effects on particle image visibility and correlation in microscopic particle image velocimetry. Experiments in Fluids, 29 (2000), 166-174.

[41] K.L. Pitts and M. Fenech, An Analytic Study on the Effect of Alginate on the Velocity Profiles of Blood in Rectangular Microchannels Using Microparticle Image Velocimetry, PLOS ONE, 8(8) (2013), e72909. doi: 10.1371/ journal.pone.0072909

[42] K.L. Pitts, R. Mehri, C. Mavriplis, and M. Fenech, Micro-particle image velocimetry measurement of blood flow: validation and analysis of data pre-processing and processing methods, Measurement Science and Technology, 23 (2012), 105302. doi:10.1088/0957-0233/23/10/105302

[43] C. Poelma, K. Van der Heiden, B. Hierck, R. Poelmann and J. Westerweel, Measurements of the wall shear stress distribution in the outflow tract of an embryonic chicken heart, Journal of the Royal Society Interface 7(42) (2009), 91-103.

[44] A. Pries, G. Kanzow and P. Gaehtgens, Microphotometric determination of hematocrit in small vessels, American Journal of Physiology: Heart and Circulatory Physiology 245(14) (1983), H167 -H177.

[45] A. Rakow, S. Simchon, L.A. Sung and S. Chien, Aggregation of red cells with membrane altered with heat treatment, Biorheology 18 (1981), 3-8.

[46] W. Reinke, P. Gaehtgens and P. Johnson, Blood viscosity in small tubes: effect of shear rate, aggregation, and sedimentation. American Journal of Physiology: Heart and Circulatory Physiology 253(3) (1987), H540 -H547.

[47] B. Salazar Vásquez, J. Martini, A. Chávez Negrete, P. Cabrales, A. Tsai and M. Intaglietta, Microvascular benefits of increasing plasma viscosity and maintaining blood viscosity: Counterintuitive experimental findings, Biorheology 46 (2009), 167-179.

[48] J. Santiago, S. Wereley, C. Meinhart, D. Beebe and R. Adrian, A particle image velocimetry system for microfluidics, Experiments in Fluids 25 (1998), 316-319.

[49] M. Savery and E. Damiano, The endothelial glycocalyx is hydrodynamically relevant in arterioles throughout the cardiac cycle, Biophysical Journal 95 (2008), 1439-1447. 
[50] J.M. Sherwood, E. Kaliviotis, J. Dusting and S. Balabani, The effect of red blood cell aggregation on velocity and celldepleted layer characteristics of blood in a bifurcating microchannel, Biomicrofluidics 6 (2012), DOI: 10.1063/1.4717755

[51] J.M. Sherwood, E. Kaliviotis, J. Dusting and S. Balabani, Hematocrit, viscosity and velocity distributions of aggregating and non-aggregating blood in a bifurcating microchannel, Biomechanics and Modelling Mechanobiology (2012), DOI: $10.1007 / \mathrm{s} 10237-012-0449-9$

[52] J.M. Sherwood, D. Holmes, E. Kaliviotis and S. Balabani, Spatial distributions of red blood cells significantly alter local haemodynamics, PLOS ONE, 9(6) (2014), doi:10.1371/journal.pone.0100473

[53] T. Shiga, K. Imaizumi, N. Harada and S. Misuzu, Kinetics of rouleaux formation using tv analyser. I. Human erythrocytes, American Journal of Physiology, Heart and Circulatory Physiology 245 (1983), H252-H258.

[54] S. Shin, M. Park, Y. Ku and J. Suh, Shear-dependent aggregation characteristics of red blood cells in a pressure-driven microfluidic channel, Clinical Hemorheology and Microcirculation 34 (2006), 353-361.

[55] M. Smith, P. Chen, C.-S. Li, S. Ramanujam and A. Cheung, Whole blood viscosity and microvascular abnormalities in Alzheimers disease, Clinical Hemorheology and Microcirculation 41 (2009), 229-239.

[56] M. Soutani, Y. Suzuki, N. Tateishi and N. Maeda, Quantitative evaluation of flow dynamics of erythrocytes in microvessels: influence of erythrocyte aggregation, American Journal of Physiology: Heart and Circulatory Physiology 268 (1995), H1959-H1965.

[57] Y. Sugii, R. Okuda, K. Okamato and H. Madarame, Velocity measurement of both red blood cells and plasma of in vitro blood flow using high-speed micro PIV technique, Measurement Science and Technology 16 (2005), 1126-1130.

[58] P. Vennemann, K. Kiger, R. Lindken, B. Groenendijk, S.Stekelenburg-de Vos, T. ten Hagen, N. Ursem, R. Poelmann, $\mathrm{J}$. Westerweel and B. Hierck, In vivo micro particle image velocimetry measurements of blood-plasma in the embryonic avian heart, Journal of Biomechanics 39 (2006), 1191-1200.

[59] P. Vennemann, R. Lindken and J. Westerweel, In vivo whole-field blood velocity measurement technique, Experiments in Fluids 42 (2007), 495-511.

[60] J. Westerweel and F. Scarano, Universal outlier detection for PIV data, Experiments in Fluids 39 (2005), 1096-1100.

[61] D. Xu, E. Kaliviotis, A. Munjiza, A. Avital, C. Ji and J. Williams, Large scale simulation of red blood cell aggregation in shear flows, Journal of Biomechanics 46(11) (2013), 1810-1817. 